\title{
Bündnisse für Arbeit: Nationale Handlungsfähigkeit im europäischen Regimewettbewerb
}

\author{
Anke Hassel
}

In den letzten Jahren lassen sich in einigen westeuropäischen Ländern wieder Formen der Konzertierung zwischen Regierungen und Tarifparteien beobachten, in denen die Beteiligten, soziale Pakte'schmieden. Der Beitrag bemüht sich um eine Erklärung des Wiederauflebens von Konzertierung auf der Makroebene unter dem Einfluss des europäischen Regimewettbewerbs. Er argumentiert, dass Regierungen zunehmend strukturelle Reformen zur Lösung des Beschäftigungsproblems suchen. Diese strukturelle Reformen sind ohne die Kooperation der Tarifparteien nahezu unmöglich. Mit der Hilfe sozialer Pakte versuchen daher Regierungen, die Zustimmung der Gewerkschaften zu einer, beschäftigungsfreundlichen'Sozial- und Tarifpolitik zu bekommen. Um diese Zustimmung zu erreichen, werden Absprachen getroffen, die den Verbänden in der Steuerund Bildungspolitik entgegenkommen. Während der Wettbewerb der Arbeits-und Sozialregime in Europa die Pakte wesentlich befördert, muss der politische Tausch zwischen Gewerkschaften und Regierungen diesen Wettbewerb nicht weiter verschärfen. Vielmehr können soziale Pakte für die europäische Koordination der Beschäftigungspolitik nützlich sein, da die Handlungsfähigkeit nationaler Verbände gestärkt wird.

\section{Einleitung}

In den letzten Jahren lassen sich in vielen westeuropäischen Ländern wieder Formen des politischen Tauschs zwischen Regierungen und Tarifparteien beobachten. ${ }^{1}$ Dieser politische Tausch nimmt dabei die Form dreiseitiger Verabredungen an, in denen die Beteiligten, soziale Pakte' über eine koordinierte Reformpolitik zur Förderung der Beschäftigung schmieden. Damit versuchen politische Akteure, das Beschäftigungsproblem über die Koordinierung von Reformmaßnahmen in verschiedenen Politikfeldern anzugehen. Nach einer Phase der bewussten Trennung von Zuständigkeiten zwischen dem Staat und Tarifparteien auf gesamtwirtschaftlicher Ebene nimmt sowohl die Rolle koordinierter Reformen als auch die Form des politischen Tauschs als Instrument an Bedeutung zu (Pochet und Fajertag 1997; Ferner und Hyman 1998; Hassel 1998; Karlhofer und Sickinger 1999; Kuntze 1998; Regini 1997 und 1999; Rhodes 1998; Schmitter/Grote 1997).

Soziale Pakte zeichnen sich durch Form und Inhalt aus. In der Form sind sie auf zentraler Ebene getroffene Vereinbarungen zur grundsätzlichen Abstimmung von Steuer-, Tarif-, So-

1 Für hilfreiche Kommentare und Diskussionen danke ich Bernhard Ebbinghaus, Werner Eichhorst, Gerda Falkner, Martin Höpner, Reiner Hoffmann, Antje Kurdelbusch, Britta Rehder, Wolfgang Streeck, Jelle Visser und Rainer Zugehör. 
zial- und Bildungspolitik. In ihrem Inhalt umfassen sie Themen, die erstens das Verhältnis der Tarifparteien zueinander neu gestalten und zweitens die Inhalte der Tarif- und Sozialpolitik den geänderten sozio-ökonomischen Bedingungen anpassen sollen. Form und Inhalt sind in einer Weise miteinander verknüpft, dass über zentrale Vereinbarungen regelmäßig auch Prozesse der Dezentralisierung von politischen Instrumenten in Gang gesetzt und kontrolliert werden sollen.

Dieser Artikel hat das Ziel, eine Erklärung für die zunehmende Entstehung sozialer Pakte ab der zweiten Hälfte der Achtzigerjahre anzubieten. Soziale Pakte werden als eine spezifische Form des politischen Tauschs zwischen Regierungen und Arbeitnehmerorganisationen zur Überwindung des Beschäftigungsproblems interpretiert. Dabei unterscheiden sich soziale Pakte von anderen Paketverhandlungen zwischen Regierungen und organisierten Interessen darin, dass in ihnen Entscheidungen auf oberster Ebene zwischen den Spitzenverbänden und der Regierung über eine Reihe verschiedener Politikfelder herbeigeführt werden. Dieser Zentralisierung der Entscheidungsfindung liegt die Hoffnung zu Grunde, über eine Umkehrung der Ausdifferenzierung einzelner Politikfelder die Problemlösungsfähigkeit der Akteure zu erhöhen. Zentralisierung kann dabei in zweierlei Hinsicht hilfreich sein: zum einen sind politikfeldübergreifende Reformen in den hochgradig interdependenten Politikfeldern Sozial- und Tarifpolitik notwendig, um die strukturellen Ursachen des Beschäftigungsproblems anzugehen. Zum anderen kann eine Zentralisierung der Entscheidungsfindung bestehende Interessendivergenzen innerhalb der Tarifparteien offen legen und den Regierungen ermöglichen, diese im Interesse einer weitreichenderen Reform auszunutzen. Interessenheterogenität innerhalb der Verbände beruht auf dem Umstand, dass in modernen Wohlfahrtsstaaten die Gruppen der Nutznießer und Kostenträger von sozialstaatlichen Regulierungen nicht mehr deckungsgleich sind. In anderen Worten: soziale Pakte dokumentieren die Versuche staatlicher Akteure, politikfeldübergreifende Reformen in prinzipiell autonomen Politikfeldern auf zentraler Ebene über die Redefinition verbandlicher Interessen zu ermöglichen.

Damit sind die sozialen Pakte der achtziger und Neunzigerjahre in Bezug auf die zu lösenden Probleme keine Neuauflage einer neo-korporatistischen Einkommenspolitik der Siebzigerjahre, wie manchmal unterstellt wird (Schmitter/Grote 1997). Die etablierten Formen korporatistischer Intermediation zwischen Staat und Tarifparteien haben mit dem Niedergang einer Politik der zentralen Nachfragesteuerung und den veränderten sozio-ökonomischen Bedingungen ihre Funktion der Einkommenspolitik verloren und werden durch die neuen Formen des politischen Tauschs nicht wiederbelebt. Vielmehr sind die in sozialen Pakten organisierte politischen Tauschhandlungen komplexer. Im Vergleich zu der neokorporatistischen Einkommenspolitik der Siebzigerjahre verfolgen die beteiligten Akteure eine erheblich größere Zahl von Reformprojekten und wenden sich in erster Linie an die Angebotsseite der Arbeitsmarktregulierung. Allerdings lassen sich im Bereich der prozeduralen Vorgehensweise Parallelen zur neo-korporatistischen Einkommenspolitik erkennen. Wie die Einkommenspolitik basieren auch soziale Pakte auf einem politischen Tausch zwischen korporativen Akteuren, in denen die Gewerkschaften für ihre Zustimmung zu politischen Reformvorhaben durch die Regierung kompensiert werden.

Der folgende Text ist in fünf Teile geteilt: nach einer Übersicht über die Reform der Beschäftigungspolitik in den Mitgliedsländern der EU und die Elemente des politischen Tauschs zwischen Regierung und Tarifparteien geht es im zweiten Abschnitt um die Frage, 
warum Regierungen zu dem Instrument eines Sozialpaktes zur Reform ihrer Sozialsysteme greifen. Aus einer institutionenorientierten Perspektive wird argumentiert, dass die Stellung, welche die Tarifparteien in dem Beschäftigungs- und Sozialsystem europäischer Wohlfahrtsstaaten einnehmen, eine strukturelle Reform der Arbeitsmärkte ohne die Kooperation der Tarifparteien nahezu unmöglich macht bzw. eine direkt gegen die Interessen der Tarifparteien gerichtete Politik erheblich höhere Kosten verursacht. Mit der Hilfe sozialer Pakte versuchen daher die politischen Akteure, die Zustimmung von den Tarifparteien zu einer ,beschäftigungsfreundlichen' Sozial- und Tarifpolitik zu bekommen. Um diese Zustimmung zu erreichen, werden Absprachen getroffen, die den Verbänden in anderen Bereichen (Steuer- und Bildungspolitik) entgegenkommen.

Im dritten und vierten Abschnitt geht es um die Frage, wie soziale Pakte zu Stande kommen. Dahinter steckt die Vermutung, dass soziale Pakte voraussetzungsreich sind und nur unter bestimmten institutionellen und politischen Bedingungen zum Abschluss kommen. Zwei Bedingungen zur Herstellung konzertierter Entscheidungen werden herausgearbeitet: erstens die Bereitschaft von Regierungen, die Federführung in zuvor weitgehend selbstkoordinierten Politikfelder wieder zu übernehmen und zweitens die Bereitschaft der Gewerkschaften, diese Federführung der Regierung im Austausch mit Mitteln zur Wiederherstellung der eigenen Handlungsfähigkeit zu akzeptieren. Für beide Bedingungen werden wiederum Faktoren benannt, die ein solches Verhalten wahrscheinlicher machen. Wo diese Bedingungen nicht gegeben sind, sind erfolgreiche Paktverhandlungen unwahrscheinlicher, oder bereits geschlossene Pakte können für einseitige verbandliche Interessen instrumentalisiert werden.

Die Möglichkeit der Instrumentalisierung sozialer Pakte bei fehlender Gemeinwohlorientierung wird im folgenden Abschnitt thematisiert. Und da soziale Pakte nicht einmalige Maßnahmen sind, sondern sich auf einen größeren Zeitraum erstrecken, stellt sich auch die Frage: Wie können staatliche Akteure diese Vereinbarungen stabilisieren, damit kurzfristige Asymmetrien nicht zu einseitigen Kündigungen einzelner Beteiligter führen? Soziale Pakte werden in der Regel unter politischen Krisenbedingungen geschlossen. Sowohl Regierungen als auch die Verbände sind der Überzeugung, dass die bestehende Beschäftigungspolitik grundsätzlich reformiert werden muss. Nach Abschluss solcher Vereinbarungen ermöglicht die Verknüpfung verschiedener Politikfelder innerhalb eines auf einen längeren Zeitraum angelegten Verhandlungsprozesses den Verbänden, ihre Entscheidungsspielräume zu vergrößern. Mit Hilfe dieser Verknüpfung werden die beteiligten Verbände in die Lage versetzt, die Prioritäten ihrer Verbändeinteressen neu zu setzen und den veränderten gesellschaftlichen Bedingungen Rechnung zu tragen. Allerdings ist in der Ungleichzeitigkeit einzelner Maßnahmen die Gefahr verborgen, dass sich einzelne Beteiligte übervorteilt sehen und aus dem Pakt wieder aussteigen. Daher lässt sich auch beobachten, dass die politischen Akteure zunehmend dafür sorgen, dass die Verbände gegen weitere Erosionstendenzen durch die Einrichtung gemeinsamer Institutionen (tripartistische Gremien, gemeinsame Fonds) und andere institutionelle Mechanismen geschützt werden.

Damit können soziale Pakte zu einer institutionellen Weiterentwicklung der Tarif- und Sozialsysteme führen, indem den Tarifparteien neue Verantwortungsbereiche und Handlungsmöglichkeiten übertragen werden. Stabile und erfolgreiche soziale Pakte stärken so die nationale Handlungsfähigkeit verbandlicher wie politischer Akteure. 


\section{Soziale Pakte in Europa}

In allen Mitgliedsstaaten der EU lassen sich seit Mitte der Achtzigerjahre parallele Anpassungsprozesse beobachten. Nationale Regierungen reagieren damit auf zwei zentrale Entwicklungen: auf den zunehmenden Wettbewerbsdruck im Rahmen der nahenden europäischen Wirtschafts- und Währungsunion und auf die Belastung der sozialen Sicherungssysteme durch anhaltende und steigende strukturelle Unterbeschäftigung. Diese Anpassungsprozesse betreffen insbesondere die institutionelle Gestaltung der Arbeitsmärkte, die Tarifpolitik und die Systeme der sozialen Sicherung. In allen Mitgliedsländern der EU finden sich einzelne Reformschritte in dem einen oder anderen Gebiet, zumeist jedoch mehrfach in allen Politikfeldern. Dabei steht unter den Bedingungen des Wettbewerbs der Arbeitsregime in den EU Mitgliedsstaaten nicht mehr die Entscheidung über die grundsätzliche Richtung der Anpassung ihrer Sozialsysteme an den europäischen Wirtschaftsraum zur Debatte. Vielmehr sind die wesentlichen institutionellen Reformelemente des Arbeitsmarktes zur Steigerung der Beschäftigungsquote - Lohnmoderation, Flexibilisierung von Arbeitsverhältnissen, Differenzierung der Entlohnung, stärkere Anreize zur Aufnahme von Arbeit - in allen EU Mitgliedsländern in der einen oder anderen Weise zu finden, unabhängig von politischen Mehrheiten und vordergründigen Differenzen zwischen den Haltungen der Tarifparteien.

Die einzelnen Elemente dieser Politik sind nicht neu, sondern bilden seit Beginn der Neunzigerjahre den Kern der beschäftigungspolitischen Diskussion auf europäischer und internationaler Ebene. Das Weißbuch der EU-Kommission, Wachstum, Wettbewerbsfähigkeit, Beschäftigung' von 1993 nennt bereits die wesentlichen Bereiche für „einschneidende Veränderungen, aber nicht bloß die Deregulierung der europäischen Arbeitsmärkte. Vielmehr ist ein neues, vernünftiges und vereinfachtes Regulierungs- und Anreizsystem nötig" (EU-Kommission 1993: 150). Auch in der Jobs Study der OECD (OECD 1997) werden die wesentlichen institutionellen Reformelemente des Arbeitsmarktes - Flexibilisierung, Differenzierung der Entlohnung, stärkere Anreize zur Aufnahme von Arbeit - aufgeführt und den OECD Mitgliedsländern zur Überwindung der Beschäftigungskrise empfohlen. In fast allen Ländern der OECD finden sich einzelne Reformschritte in dem einen oder anderen Gebiet.

Es gibt in diesen Bemühungen, nationale Tarif- und Sozialsysteme an neue Umweltbedingungen anzupassen, jedoch maßgebliche Unterschiede in der Rolle der Tarifparteien. Für die Regierungen scheint es im Wesentlichen zwei Wege zu geben, ihre Ziele in der Beschäftigungspolitik zu erreichen: den Weg der Kooperation mit den Tarifparteien oder den Weg der einseitigen Dezentralisierung und Deregulierung nationaler Beschäftigungssysteme. Die Länder, in denen Tarifsysteme gegen den Widerstand der Gewerkschaften dezentralisiert wurden, haben diesen Prozess weitgehend in den Achtzigerjahren abgeschlossen. In den betroffenen Ländern, insbesondere Großbritannien und Frankreich, sind auch unter den Linksregierungen der Neunzigerjahre keine bedeutsamen Kooperationen mit den Gewerkschaften zu beobachten. ${ }^{2}$ In den Ländern, in denen Regierungen den Dialog mit Arbeitgebern und Gewerkschaften in den Achtzigerjahren nicht aufgegeben haben, kann man

2 Eine Ausnahme ist der von der Jospin-Regierung initiierte dreiseitige Gipfel in Matignon zur Umsetzung des Arbeitszeitgesetzes im Jahr 1997, der jedoch mit dem Auszug der Arbeitgeber aus den Verhandlungen endete (siehe Ebbinghaus/Hassel 2000). 
ab Mitte der Achtzigerjahre hingegen zunehmende Kooperationsbemühungen zwischen den Regierungen und Tarifparteien beobachten. In einigen Ländern (Dänemark, Österreich) bauten diese Bemühungen auf bestehenden Kooperationen auf, ohne explizit zu neuen Vereinbarungen zu führen. In einer ganzen Reihe anderer Länder führten diese Bemühungen jedoch zum Abschluss von neuen Kooperationsvereinbarungen.

Ob mit Hilfe sozialer Pakte oder gegen den Widerstand der Tarifparteien - die institutionelle Gestaltung der Arbeitsmärkte, die Tarifpolitik und die Systeme der sozialen Sicherung ist überall Gegenstand von Reformen. ${ }^{3}$ In der Bewertung durch die OECD wie auch den ,Benchmarking' Bemühungen der EU Kommission werden regelmäßig zwei Gruppen von Ländern ausgezeichnet. Erstens werden besonders von der OECD stets solche Länder als vorbildlich beschrieben, die ihre Arbeitsmarktreformen mit besonders drastischen Maßnahmen gegenüber den traditionellen Formen der Regulierung der Arbeitsmärkte durchgeführt haben. Das trifft auf die angelsächsischen Länder mit Ausnahme Irlands zu. Und zweitens führen sowohl OECD als auch die EU Kommission auch zunehmend die Länder als positive Reformbeispiele an, in denen Sozialpakte zur Arbeitsmarktreform geschlossen wurden (wie etwa die Niederlande und Irland). ${ }^{4}$

Der Unterschied zwischen der angelsächsischen Variante der institutionellen Reform des Arbeitsmarktes und den Reformen des Arbeitsmarktes im Rahmen sozialer Pakte besteht darin, dass soziale Pakte eine Verknüpfung von deregulierenden und Kosten senkenden Maßnahmen mit ausgleichenden Instrumenten durch die öffentliche Hand herzustellen versuchen. Auch in den Vereinbarungen zu Sozialpakten spielen ,Kostensenkung', ,Anreizstrukturen zur Arbeitsaufnahme' und ,Flexibilisierung' als die beherrschenden Themenbereiche die entscheidende Rolle. Es geht daher nicht um einen grundsätzlich anderen Ansatz in der Reformpolitik, sondern in sozialen Pakten wird die gesellschaftliche Akzeptanz für Reformen in der Sozial- und Tarifpolitik durch die Einbindung der Tarifparteien ermöglicht.

Stabile soziale Pakte findet man seit Mitte der Achtzigerjahre in den Niederlanden, Italien, Irland und Finnland. In anderen Ländern wurden ähnliche Vereinbarungen zwischen Regierungen und Tarifparteien angestrebt, jedoch nicht von allen Akteuren unterstützt bzw. umgesetzt (vgl. Tabelle 1). In Schweden haben die Tarifparteien im Oktober 1998 nicht-öffentliche Gespräche über einen Wachstumspakt aufgenommen. Diese Verhandlungen scheiterten im Frühjahr 1999 an dem Widerstand der Arbeitergewerkschaft. In Belgien wurde ein sozialer Pakt 1993 und 1996 von der Regierung vorgeschlagen und scheiterte an dem Widerstand der Gewerkschaften. Die wesentlichen Elemente des Paktes wurden daraufhin von der Regierung auf gesetzlichem Wege umgesetzt. In Portugal mangelte es bei der Umsetzung des Sozialpakts an der Unterstützung der größten Gewerkschaft CGTP. In Griechenland (1997) und Spanien (1997) wurden Sozialpakte zwischen den Gewerkschaften und Regierungen abgeschlossen, jedoch von den Arbeitgebern abgelehnt. Auch in Deutschland kam ein von den Gewerkschaften angestrebtes Bündnis für Arbeit und Stand-

3 Siehe im Überblick dazu die Berichte der OECD ,Implementing the Jobs Study' (OECD 1997) sowie die Zusammenfassung der nationalen Aktionspläne an die EU Kommission in ,Joint Action Programm' (EU-Kommission 1998a) und die Zusammenfassung in den Joint Employment Reports 1997 und 1998 (EU-Kommission 1997 und EU-Kommission 1998b).

4 Analog auch der Monatsbericht der Deutschen Bundesbank im Oktober 1999 (Deutsche Bundesbank 1999). 
Tabelle 1: Soziale Pakte in Europa

\begin{tabular}{|c|c|c|}
\hline Länder & Jahr & Name \\
\hline \multicolumn{3}{|c|}{ Erfolgreiche Soziale Pakte } \\
\hline \multirow[t]{4}{*}{ Niederlande } & 1982 & $\begin{array}{l}\text { Abkommen über ,allgemeinverbindliche Empfehlungen zu Fragen der Be- } \\
\text { schäftigungspolitik' (Wassenaar) }\end{array}$ \\
\hline & 1990 & Mehr Beschäftigung für ethnische Minderheiten \\
\hline & 1993 & Ein neuer Kurs: Agenda für Tarifverhandlungen 1994 \\
\hline & 1997 & Agenda 2002 \\
\hline \multirow[t]{4}{*}{ Irland } & 1987 & Programme for National Recovery \\
\hline & 1990 & Programme for Economic and Social Progress \\
\hline & 1994 & Programme for Competitiveness and Work \\
\hline & 1997 & Partnership 2000 for Inclusion, Employment and Competitiveness \\
\hline \multirow[t]{4}{*}{ Italien } & 1992 & Dreiseitige Vereinbarung zur Abschaffung der Scala Mobile \\
\hline & 1993 & Ciampi Protokoll: Abkommen über Arbeitskosten vom 23. Juli 1993 \\
\hline & 1996 & Beschäftigungspakt (Accordo per il Lavoro) \\
\hline & 1998 & Sozialer Pakt für Wachstum und Beschäftigung \\
\hline Dänemark & 1987 & Sozialpartnerschaftliche Absprache \\
\hline \multirow[t]{3}{*}{ Finnland } & 1991 & Stabilitätspaket \\
\hline & 1995 & Sozialpakt \\
\hline & 1998 & Sozialkontrakt \\
\hline \multicolumn{3}{|c|}{ Instabile Vereinbarungen } \\
\hline Griechenland & 1997 & $\begin{array}{l}\text { Pakt für Wettbewerbsfähigkeit (ohne Zustimmung der Klein- und Mittleren } \\
\text { Unternehmen) }\end{array}$ \\
\hline \multirow[t]{2}{*}{ Spanien } & 1994 & Toledo Pact (ohne Zustimmung der Arbeitgeber) \\
\hline & 1997 & Vereinbarung der Tarifparteien \\
\hline \multirow[t]{2}{*}{ Portugal } & 1996 & $\begin{array}{l}\text { Kurzfristiger Dreiseitiger Vertrag (Acordo de Concertado Social de Curto } \\
\text { Prazo) (nicht von CGTP unterzeichnet) }\end{array}$ \\
\hline & 1997 & Strategischer Sozialer Pakt (Acordo de Concertacao Estrategica) \\
\hline \multirow[t]{3}{*}{ Belgien } & 1993 & Globaler Plan (gescheitert) \\
\hline & 1996 & $\begin{array}{l}\text { Verhandlungen über einen Vertrag über die Zukunft der Beschäftigung (ge- } \\
\text { scheitert) }\end{array}$ \\
\hline & 1998 & Intersektoraler Tarifvertrag (nach gesetzlicher Intervention) \\
\hline \multirow[t]{2}{*}{ Deutschland } & 1996 & Bündnis für Arbeit und Standortsicherung (gescheitert) \\
\hline & 1998 & Bündnis für Arbeit, Ausbildung und Wettbewerbsfähigkeit \\
\hline Schweden & 1999 & Wachstumspakt (allians för tillväxt) gescheitert. \\
\hline
\end{tabular}

ortsicherung Anfang 1996 nicht zu Stande (Bispinck 1997); es wurde dann aber nach der Bundestagswahl 1998 zu dem wichtigsten politischen Projekt der neuen Regierungskoalition. Der erste Schritt zu einem erneuten Bündnis für Arbeit wurde am 07. Dezember 1998 durch die Verabschiedung einer gemeinsamen Erklärung von Regierungs- und Verbandsvertretern getan.

Stabile Sozialpakte zeichnen sich dadurch aus, dass erstens die auf zentraler Ebene getroffenen Vereinbarungen von allen wesentlichen Akteuren getragen und zweitens in regelmä- 
ßigen Abständen neu abgeschlossen werden. Trotz bestehender Konflikte im weiteren Verlauf der Sozialpakte haben Momente der Unzufriedenheit bislang noch in keinem Fall zu einer Aufkündigung der bereits getroffenen Vereinbarungen geführt. Das „loyale Verhalten“ der Akteure (so Sergio Cofferati, Generalsekretär der CGIL) wurde durch kurzfristige Tarifkonflikte nicht aufgebrochen (Hassel 1998: 634).

Die zwischen Regierungen und Tarifparteien abgeschlossenen Vereinbarungen beziehen sich auf die institutionellen Ursachenfaktoren des Beschäftigungsproblems und umfassen ,beschäftigungsfördernde' Reformen in der Sozial- und Tarifpolitik. Die wichtigsten Themenbereiche sind dabei Kostensenkung und Flexibilisierung, die mit Kompensationsmaßnahmen versehen werden: Arbeitskosten sollen zur Verbesserung der Wettbewerbsfähigkeit oder zur Beschäftigungsexpansion im Dienstleistungssektor gesenkt werden, ohne dass Arbeitnehmer notwendigerweise an Einkommen verlieren. Arbeitsmärkte sollen flexibilisiert und dynamisiert werden, ohne dass Arbeitnehmer von den neuen Flexibilitätserfordernissen überfordert werden, bzw. Arbeitnehmer sollen in die Lage versetzt werden, diesen Erfordernissen gerecht $\mathrm{zu}$ werden.

In dieser stilisierten Form finden sich die Verabredungen nur selten. In der Regel erfolgen die in den Pakten vereinbarten Einzelmaßnahmen in kleinen Schritten, die auch im Abstand von mehreren Jahren neu entschieden werden. Italien ist jedoch ein Beispiel, wo die Austauschmechanismen des Paktes besonders deutlich werden. In dem Pakt für Entwicklung und Beschäftigung vom Dezember 1998 wurde eine Reduzierung des Steuersatzes für Jahreseinkommen zwischen 15 und 30 Mio. Lire von 27 auf 26 Prozent festgelegt. Die Regierung versprach zudem, in den nächsten drei Jahren 1,6 Billionen Lire in berufliche Bildungsmaßnahmen zu investieren. Auf Forderung der Unternehmer hin hat die Regierung zugestimmt, dass Abgaben auf betrieblich vereinbarte Lohnbestandteile um einen Prozentpunkt reduziert werden. Bis zum Jahr 2003 sollen die Lohnkosten um 1,25 Prozent reduziert werden, indem bestimmte Teile der Lohnnebenkosten abgeschafft oder verschoben werden sollen. Zudem verpflichtet sich die Regierung, bürokratische Hürden für die Unternehmen abzubauen und Anreize zu entwickeln, um irreguläre Beschäftigungsverhältnisse in reguläre umzuwandeln (Telljohann 1999).

Stellt man die Interessen der einzelnen Beteiligten gegenüber, so findet man neben den traditionellen gegensätzlichen Interessen - etwa in den Fragen des Lohns und der Arbeitszeit - durchaus Themen, bei denen eine gemeinsame Interessenlage hergestellt werden kann. Im Gesamtpaket haben alle beteiligten Seiten relative Zugewinne wie Zugeständnisse zu verzeichnen. Im Interesse der Gewerkschaften waren Arbeitszeitverkürzungen, Arbeitsbeschaffungsprogramme sowie Steuererleichterungen; ihre Zugeständnisse lagen in Fragen der Flexibilisierung von Arbeitsverhältnissen, Dezentralisierung von Tarifpolitik und natürlich in zurückhaltenden Lohnforderungen. Dem Staat wurden reduzierte Belastungen für Ausgaben der sozialen Sicherung und eine Kontrolle über die Kostenentwicklung im öffentlichen Dienst in Aussicht gestellt; zugleich wurden den öffentlichen Haushalten aber höhere Ausgaben in Bereichen der öffentlichen Subventionierung von Beschäftigung und Bildungspolitik zugemutet. Für die Arbeitgeber ermöglichten die Vereinbarungen eine Flexibilisierung und Dezentralisierung der Tarifpolitik, Steuererleichterungen, Reformen und Kostenentlastungen der sozialen Sicherungssysteme und eine moderate Lohnentwicklung; sie mussten Positionen in der Frage der Arbeitszeiten aufgeben. Durch die Einbindung in dreiseitige Vereinbarungen mussten sich die Arbeitgeber zudem von Versuchen distanzieren, ihre ge- 
stiegene Marktmacht gegenüber den Gewerkschaften einseitig auszunutzen und arbeitsrechtliche und tarifpolitische Absprachen zu umgehen.

Damit lag die Mehrheit der Tauschgeschäfte im Verhältnis zwischen Staat und Gewerkschaften und quer zu den bestehenden Entscheidungsmechanismen der Politikfelder. Sie waren nur durch die Zusammenführung der Akteure in den Bereichen Tarifpolitik und Sozialpolitik möglich. Nur durch die Verbindung zwischen Instrumenten der öffentlichen Hand und der Tarifpolitik konnten die Koppelgeschäfte erfolgen: Die Kompensation von Lohnzurückhaltung durch die Steuerentlastung von Arbeitnehmereinkommen und die Entkoppelung der Tarifentwicklung im öffentlichen Dienst mit der Steuerung öffentlicher Defizite erforderten politikfeldübergreifende Abstimmungen und Akteure. Reine Zugewinne der Tarifparteien auf Kosten der öffentlichen Hand bestanden in Verbesserungen der beruflichen Bildung, Steuererleichterungen und Vorruhestandsregelungen.

Die Lohnzurückhaltung der Gewerkschaften, als wichtigstes Instrument der Kostensenkung, stand in allen sozialen Pakten sowohl zeitlich als auch von ihrer Bedeutung her an erster Stelle. Die Gewerkschaften erklärten sich bereit, niedrige Lohntarifverträge langfristig abzuschließen, um die Wettbewerbsposition ihres Landes zu verbessern oder zumindest nicht zu gefährden. Die Lohnentwicklung wurde entweder durch Lohnleitlinien über einen Zeitraum von zwei bis drei Jahren für die gesamte Volkswirtschaft festgelegt (Irland), oder man einigte sich auf eine Formel, dass sie die Preissteigerung nicht wesentlich übersteigt (Italien) oder sich an der Lohnentwicklung in anderen europäischen Ländern orientiert (Belgien, Niederlande). In Portugal wurde in dem strategischen Abkommen 1996 eine feste Lohnleitlinie und für die folgenden Jahre die Faustregel vereinbart, dass Lohnerhöhungen nur knapp die Hälfte der Produktivitätszuwächse betragen durften. Formen der automatischen Indexierung von Löhnen an die Preissteigerung (wie etwa in Belgien und Italien) wurden wieder aufgehoben, um Lohnentwicklungen besser steuern zu können. ${ }^{5}$

Den Gewerkschaften wurde von Regierungsseite und Arbeitgeberseite in verschiedener Weise entgegengekommen, um Lohnzurückhaltungen zu erleichtern. In der Steuerpolitik wurde in Irland und Portugal verabredet, mit Steuersenkungen Arbeitnehmereinkommen zu entlasten und dadurch Lohnzurückhaltung zu belohnen. In den Niederlanden wurden Niedrigeinkommen steuerlich begünstigt. In anderen Ländern (Niederlande und Portugal) zeigten sich die Arbeitgeber bereit, in Verhandlungen über Arbeitszeitverkürzung einzutreten. Dies führte zu weiter gehenden kollektiven Arbeitszeitverkürzungen. Nach Abschluss der Vereinbarung von Wassenaar konnte in den Niederlanden im Laufe der Achtzigerjahre die Arbeitszeit schrittweise auf 36 Stunden verkürzt werden. In Portugal wurde 1995 von

5 In der Lohnpolitik finden sich ähnliche Absprachen auch für Dänemark, Schweden und Finnland. In Dänemark einigten sich Gewerkschaften, Arbeitgeber und Regierung schon 1987 darauf, dass die dänische Lohn- und Gehaltsentwicklung nicht über dem Niveau des Auslands liegen sollte (,sozialpartnerschaftliche Absprache'). In Schweden wurde die Einführung einer Europa-Norm vorgeschlagen, wonach die schwedischen Löhne nicht schneller als im europäischen Durchschnitt wachsen sollten. Diese Norm wurde mittlerweile auch von weiten Teilen der schwedischen Gewerkschaften akzeptiert (Schulten 1998). In Finnland wurde 1991 zunächst ein Stabilisierungspakt mit Lohnstopp vereinbart. 1995 und 1997 wurden Sozialkontrakte abgeschlossen, in denen niedrige Lohnabschlüsse festgeschrieben wurden (Kuntze 1998: $30)$. 
der sozialistischen Partei die 40-Stundenwoche eingeführt, die später innerhalb des strategischen Pakts mit Maßnahmen zur Flexibilisierung der Arbeitszeit verknüpft wurde. ${ }^{6}$

Auf der Kostenseite kam zudem die Kostenentwicklung des öffentlichen Sektors hinzu. In allen sozialen Pakten war die Reform der Tarifverhandlungssysteme im öffentlichen Dienst ein eigenständiger Gegenstand. In Irland, den Niederlanden und Italien dienten die Reformen der Lohnfindung im öffentlichen Dienst der Abkoppelung der Lohnentwicklung im öffentlichen Dienst von der Lohnentwicklung in der Privatwirtschaft. Für die Regierungen war sowohl die allgemeine Senkung der Zuwachsraten der Lohnsumme im öffentlichen Dienst von Bedeutung als auch die Frage der Steuerbarkeit von Lohnentwicklungen im öffentlichen Dienst.

Zur Kostensenkung gehörte auch eine stärkere Differenzierung von Lohnentwicklungen nach der Leistungsfähigkeit einzelner Unternehmen und Branchen. Dafür war eine stärkere Dezentralisierung der Lohnfindung erforderlich. Trotz zentral festgelegter Prinzipien der Lohnentwicklung nahm die Bedeutung von Tarifverhandlungen auf der Branchen- und Unternehmensebene zu. Für die Koordinierung zwischen zentralen Lohnleitlinien und dezentralen Verhandlungen entwickelten die Tarifparteien Regeln und Prozeduren, mit denen zentrale Vorgaben über allgemeine Lohnentwicklungen mit dezentralen Verhandlungen über die Vergütung unternehmensspezifischer Produktivitätsentwicklungen und der Schaffung arbeitsplatzbezogener Anreizstrukturen kombiniert werden können. In Italien wurde nach der ersten Vereinbarung zur Abschaffung der Lohnindexierung (Scala Mobile) im Jahr 1992 im zweiten Schritt das Tarifverhandlungssystem neu reguliert und systematisiert. Es wurden zwei Ebenen der Tarifverhandlungen mit jeweils eigenen Verhandlungsmodi eingerichtet: zentrale Verhandlungen zur Steuerung der allgemeinen Lohnentwicklung und Branchen- und Betriebsverhandlungen zur Festlegung relativer Lohnniveaus. Auch in den Niederlande hat die Vereinbarung von Wassenaar (1982) zu einer, organisierten Dezentralisierung' (Traxler 1995) des Tarifverhandlungssystems geführt. In den irischen Vereinbarungen wurde den zentralen Lohnerhöhungen die Möglichkeit dezentraler Verhandlungen über produktivitätsorientierte Lohnsteigerungen unter eng definierten Bedingungen (Fristen und Obergrenzen) hinzugefügt.

Im Bereich der Flexibilisierung der Arbeitsmärkte finden sich Maßnahmen zum Ausbau der Leiharbeit und der befristeten Beschäftigung (Niederlande) und Maßnahmen zur Senkung der Lohnnebenkosten (Belgien, Irland) wie auch allgemein zur Senkung der Arbeitskosten von Langzeitarbeitslosen und Geringqualifizierten (Dänemark, Italien, Portugal). Arbeitsrechtliche Maßnahmen zur Aktivierung des Arbeitsmarktes gab es insbesondere in Ländern mit hoher Jugendarbeitslosigkeit und starken Senioritätsrechten für Beschäftigte. In Italien wurde die Leiharbeit erstmals legalisiert, von der man sich eine flexibilisierende Wirkung erhoffte. Ebenso gehörte die Lockerung des Kündigungsschutzes in Portugal zu den flexibilisierenden Elementen des Sozialen Paktes. Im Hinblick auf die Anreizstruktur zur Arbeitsaufnahme wurde ein zunehmender Druck auf Arbeitslose zur Arbeitsaufnahme ausgeübt, der mit der Bereitstellung von Arbeitsmöglichkeiten verbunden wurde. In allen Reformen im Rahmen sozialer Pakte lässt sich die Frage erkennen, wie Transferleistungen

6 Allerdings wurde im Anschluss an das Gesetz zwar die Arbeitszeitverkürzung, nicht jedoch die Flexibilisierung umgesetzt, da die CGTP - als die nicht an dem Pakt beteiligte Gewerkschaft in den Unternehmen Tarifverträge abschloss, die keine Arbeitszeitflexibilisierung erlaubten. 
an Nichterwerbstätige durch die öffentliche Hand in eine Bereitstellung von Arbeitsmöglichkeiten und Anreize zur Arbeitsaufnahme umgewandelt werden können.

Der Druck zur Arbeitsaufnahme wurde auch durch die Kürzung von Transferansprüchen erhöht. In Italien und Holland wurden Rentenansprüche für Personen unterhalb des Rentenalters eingeschränkt. In beiden Ländern gab es eine zunehmende Belastung der Rentensysteme durch ,jüngere' Rentenempfänger (in den Niederlanden durch den stets steigenden Anteil von Empfängern von Erwerbsunfähigkeitsrenten; in Italien durch Senioritätsrenten, die jeder Arbeitnehmer nach 35 Jahren Beschäftigung erhalten konnte). Die Rentenreformen 1993 in den Niederlanden und 1995 in Italien reduzierten sowohl Ansprüche als auch Leistungen drastisch. In beiden Ländern wurden durch die hohen Renten- und Sozialausgaben die öffentlichen Haushalte stark belastet.

Zugleich wurden jedoch auch neue Arbeitsbeschaffungsprogramme für ausgegrenzte Gruppen auf dem Arbeitsmarkt entwickelt. In Irland und den Niederlanden wurden Arbeitserfahrungsprogramme in der Form von Praktika für Langzeitarbeitslose eingeführt. In den Niederlanden gab es zudem zielgruppenorientierte Programme wie etwa die Vereinbarung zur Schaffung von Arbeitsplätzen für ethnische Minderheiten im Jahr 1990. Zugleich erhielten in Italien und Irland Unternehmen steuerliche Anreize zur Schaffung von Arbeitsplätzen. Steuererleichterungen für Unternehmen in Italien sollten die Integration von jungen Menschen (unter 32), von Langzeitarbeitslosen und Behinderten ins Erwerbsleben fördern. In Italien und in Portugal wurden Maßnahmen zur Verbesserung der Lehrlingsausbildung ergriffen. In Portugal wurde ein individuelles Recht auf eine mindestens einjährige Ausbildung für alle Jugendlichen geschaffen. Zudem wurde in Italien die allgemeine Schulpflicht verlängert.

\section{Das Beschäftigungsproblem: Interdependente Politikfelder und verflochtene Akteurskonstellationen}

Warum Regierungen auf zentrale Vereinbarungen mit Tarifparteien zur Lösung des Beschäftigungsproblems zurückgreifen, kann man erklären, wenn man die Politikfelder und Akteurskonstellationen in der Beschäftigungspolitik betrachtet. Die Fähigkeit nationaler Regierungen, das Beschäftigungsproblem allein zu bearbeiten, ist in dreifacher Weise beschränkt. Die erste Beschränkung liegt in der Komplexität der Wirkungszusammenhänge auf dem Arbeitsmarkt. Die Entwicklung der Beschäftigung hängt von einem Bündel unterschiedlicher Faktoren ab, von denen einige konjunkturell, andere strukturell bestimmt sind. Die genaue Bewertung der einzelnen Faktoren für die Beschäftigungsentwicklung ist im wissenschaftlichen Diskurs umstritten. Zu der wissenschaftlichen Kontroverse kommt hin$\mathrm{zu}$, dass die Interpretation des Beschäftigungsproblems nicht verteilungsneutral ist. Die relative Bedeutung konjunktureller und struktureller Faktoren und die damit verbundenen Lösungsvorschläge betreffen verschiedene Gruppen in unterschiedlicher Weise. Sie ist interessengebunden und daher auch im politischen Prozess kontrovers.

Die zweite Beschränkung der Handlungsfähigkeit nationaler Regierungen liegt in der Interdependenz verschiedener Politikfelder. Der Arbeitsmarkt wird von verschiedenen Politikfeldern beeinflusst, von denen die Fiskalpolitik, die Steuerpolitik, die Tarifpolitik und die Sozialpolitik zu den bedeutendsten zählen. Diese verschiedenen Politikfelder haben un- 
terschiedliche Effekte auf das Beschäftigungsproblem, die sich gegenseitig unterstützen, aber auch aufheben oder unterminieren können. Hinzu kommt, dass die maßgeblichen Akteure in diesen wichtigen Politikfeldern in erster Linie andere Ziele als die Förderung der Beschäftigung verfolgen. Das für das Beschäftigungsproblem eigens geschaffene Politikfeld Arbeitsmarktpolitik hat hingegen im Vergleich zu den vier erst genannten einen eher geringen Einfluss auf die Beschäftigungsentwicklung.

Die Interdependenz der einzelnen Politikfelder stellt hohe Anforderungen an die Koordinierung von Reformen. Dabei macht das Erbe eines hohen Niveaus an funktioneller Interdependenz von Beschäftigungs- und Wohlfahrtssystemen in den westlichen Nachkriegsökonomien es auf der Ebene der politischen Steuerung noch schwieriger, diese an die neuen Arbeitsmarkt- und Familienstrukturen anzupassen. Autonome Strukturveränderungen im Beschäftigungssystem beeinflussen die Effizienz des Wohlfahrtssystems und umgekehrt (Freeman 1995: 17). Unkoordinierte Reformen in ausdifferenzierten und zugleich interdependenten Politikfeldern können sich konterkarieren. Zum Beispiel können Kürzungen von Transferleistungen ohne eine Belebung des Arbeitsmarktes zu größerer Armut führen, die wiederum von anderen sozialen Sicherungssystemen aufgefangen werden muss. Öffentlich geförderte Beschäftigungsprogramme können Arbeitsverhältnisse in privaten Unternehmen verdrängen, oder zu Mitnahmeeffekten führen. Andere Formen der Subventionierung von Familieneinkommen durch Einkommenssubventionen können zu Sozialhilfefallen mutieren, da sich die Arbeitsaufnahme nicht mehr lohnt.

Gleichzeitig können Reformen in einem Politikfeld nur schwer mit anderen Reformen koordiniert werden, da in den einzelnen Politikfeldern sowohl unterschiedliche Akteurskonstellationen als auch unterschiedliche institutionelle Bedingungen im Entscheidungsprozess vorliegen. Dabei sind die wichtigsten Akteure in der Beschäftigungspolitik neben den politischen Akteuren die Tarifparteien. In den meisten westeuropäischen Ländern sind die Tarifparteien nicht nur an der zentralen Rolle der Lohn - und Arbeitsmarktgestaltung über die Tarifpolitik beteiligt, sondern haben durch die Verwaltungsorgane der Sozialversicherungsträger auch in der Sozialpolitik wesentliche Einflussmöglichkeiten und Vetorechte. In diesen unterschiedlichen Arenen haben sie wiederum unterschiedliche Funktionen zu vertreten. Auf Grund der Unterschiede in den Formen der Entscheidungsfindung - the structure of the game (Immergut 1992) - können auch die gleichen Akteure in verschiedenen Arenen verschiedene - manchmal auch konfligierende - Positionen einnehmen und verfolgen.

Diese Unterschiede in Funktionen und Interessenlagen können dazu führen, dass Reformen in einem Politikfeld von einer bestimmten Interessenkonstellation blockiert werden, die in anderen Feldern so nicht besteht. In Deutschland können die Gewerkschaften z.B. Transferleistungen für Lohnersatz über die Bundesanstalt für Arbeit beeinflussen, nicht jedoch Leistungen der Sozialhilfe. Oder die Tarifparteien können ein - aus ihrer Sicht - nachrangiges Politikfeld dafür benutzen, Erfolge in einem anderen zu erreichen.

Drittens sind staatliche Maßnahmen zur Beschäftigungsförderung dadurch eingeschränkt, dass sich die einzelnen zentralen Politikfelder im Laufe der Zeit zunehmend sektoral ausdifferenziert haben und innerhalb einzelner Regierungen unterschiedlichen Fachzuständigkeiten unterliegen. Die Politikfelder an sich weisen wiederum eine hohe Varianz in der Verschränkung zwischen staatlichen Akteuren und Verhandlungssystemen mit korporativen und kollektiven Akteuren auf. Wir wissen aus der Literatur über die (Rück-)Ent- 
wicklung der Wohlfahrtsstaaten (Retrenchment), dass der Rückzug des Wohlfahrtsstaates anderen Regeln folgt als sein Ausbau. Paul Pierson argumentiert anhand der Beispiele der USA und Großbritannien, dass Wohlfahrtsstaaten ihr eigenes Klientel produziert haben, die es erfolgreich vermögen, bestimmte Anpassungsreformen an neue Beschäftigungsstrukturen zu verhindern (Pierson 1994).

Damit besteht das Dilemma einer staatlichen Beschäftigungspolitik darin, dass sich staatliche Handlungsmöglichkeiten in einer komplexen Verflechtung mit Formen horizontaler Selbstregulierung durch korporative und kollektive Akteure in einer Reihe unterschiedlicher und sektoral ausdifferenzierter Politikfelder befinden, in denen der Staat nicht nur sachlich sondern auch zeitlich koordiniert tätig werden müsste. Die Steuerungsinstrumente sind zumindest in den Teilsystemen Tarifpolitik, Arbeitsmarktpolitik und soziale Sicherung von komplexer Natur; die Akteurskonstellationen sind auf Grund der nationalspezifischen Evolution kollektiver Akteure mehrfach verflochten, wobei die Tarifparteien in der Regel sowohl in der staatsfreien Selbstregulierung in der Tarifpolitik wie auch in der Sozial- und Arbeitsmarktverwaltung eine wichtige Rolle spielen.

Dieses vielfach verflochtene Zusammenwirken von Verhandlungssystemen und Akteurskonstellationen in den verschiedenen Politikfeldern der Beschäftigungspolitik behindert so die Möglichkeit des Staates, strukturelle Reformen in der Tarif- und Sozialpolitik autonom durchzuführen. Die dadurch entstehenden institutionellen Blockaden allein erklären jedoch noch nicht den Rückgriff der Regierungen auf Konzertierung zur Lösung des Beschäftigungsproblems, sondern lediglich das Scheitern größerer Reformvorhaben in der Tarif- und Sozialpolitik in der Vergangenheit.

Warum lassen sich die bestehenden Blockaden durch einen politischen Tausch zwischen Regierungen und Tarifparteien auflösen? Warum lassen sich Tarifparteien, die nicht gemeinwohlgebunden sind, auf zentrale Verhandlungssysteme ein, deren Ziel es ist, sie zumindest partiell von der Verfolgung ihrer Verbandsinteressen zu Gunsten des Gemeinwohls abzubringen? Zudem werden im Rahmen sozialer Pakte sowohl regulative als auch distributive Fragen behandelt und scheinbar auch entschieden. Da man in der Regel davon ausgehen muss, dass auf Umverteilung zielende Maßnahmen in Verhandlungssystemen, die auf freiwillige Zustimmung angewiesen sind, in der Regel nicht konsensfähig sind (Scharpf 1991a: 629), stellt sich auch die Frage, wie die Zustimmung zu solchen Verhandlungsergebnissen gesichert werden kann.

Im Folgenden sollen zwei zentrale Bedingungen zur Herstellung konzertierter Entscheidungsfindungen zur Reform von Arbeitsmarkt- und Wohlfahrtssystemen herausgearbeitet werden: die Herstellung von hierarchischen Verhandlungssystemen durch die Intervention von Regierungen und die Neupositionierung der Gewerkschaften in einem stärker hierarchisch strukturierten Entscheidungssystem. Regierungen müssen bereit sein, die Federführung in zuvor weitgehend selbstkoordinierten Politikfelder wieder zu übernehmen und Gewerkschaften müssen diese Federführung der Regierung zur Wiederherstellung der eigenen Handlungsfähigkeit akzeptieren. Wo beides nicht der Fall ist, können Paktverhandlungen entweder nicht zu Stande kommen, oder bereits geschlossene Pakte können für verbandliche Interessen instrumentalisiert werden. 


\section{Die Rolle der Regierung: Herstellung von Hierarchie in der Beschäftigungspolitik}

In vielen Bereichen selbstkoordinierter Verhandlungssysteme wird die Position und die Verhandlungsstärke einzelner korporativer Akteure durch die Regelsetzung der Regierung bestimmt (Scharpf 1993: 146). Regierungen haben eine Vielzahl von Einflussmöglichkeiten auf die Tarifvertragsparteien, ohne sich direkt mit ihnen an den Verhandlungstisch zu setzen. Erstens kann der Staat über seine Möglichkeiten der steuerlichen und sozialpolitischen Be- und Entlastung von Arbeitnehmern und Unternehmen die Tarifpolitik indirekt beeinflussen. Dies gilt für die Steuerbelastung von Einkommen im allgemeinen, aber auch insbesondere für die einkommensabhängigen Beitragsleistungen an die Systeme der sozialen Sicherung. Diese liegen nicht im Entscheidungsbereich der Tarifparteien, beeinflussen jedoch die Tarifpolitik insofern, als dass steigende Belastungen der Arbeitnehmereinkommen zu steigenden Erwartungen an die Gehaltsentwicklung führen. Umgekehrt kann die steuerliche Entlastung von Arbeitnehmereinkommen und Unternehmen auch größere Spielräume in der Gestaltung von Tarifforderung ermöglichen.

Zweitens kann der Staat die Tarifparteien auf Kosten der Sozialversicherungssysteme entlasten. Zum Beispiel finden sich Allianzen der Tarifparteien bei der Nutzung der Sozialkassen bei den Regelungen des Vorruhestands. Der Vorruhestand wurde regelmäßig als ein Instrument benutzt, das den Unternehmen die Umstrukturierung von Produktionsprozessen erleichterte, wie auch den Gewerkschaften ihre Zustimmung zu Rationalisierung und Modernisierung ermöglichte, in dem ältere Arbeitnehmer und Arbeitnehmer mit verminderter Leistungsfähigkeit nicht von Entlassung bedroht wurden, sondern in den vorgezogenen Ruhestand geschickt wurden. Solche gemeinsamen Strategien der Tarifparteien zur Externalisierung (Oppen 1997) von Rationalisierungskosten, lassen sich eher dort finden, wo die Tarifparteien in die Sozialverwaltung involviert sind. ${ }^{7}$ Da die Systeme der sozialen Sicherung, aus denen Vorruhestandsprogramme finanziert werden, der staatlichen Aufsicht unterliegen, kann die Regierung die Verfügung über solche Fonds an Bedingungen knüpfen, bzw. ihre Nutzung durch die Tarifparteien entweder erleichtern oder erschweren.

Zudem kann der Staat durch die Rahmensetzung der Tarifvertragssysteme das relative Machtgleichgewicht zwischen den Tarifparteien durch die Änderung der Rahmenbedingungen modifizieren. Zum Beispiel dienen alle Standards der sozialen Sicherung wie auch die materiellen Regulierungen des Arbeitsrechts als ein Mindeststandard von Rechten der

7 Externalisierungsstrategien finden ihre Grenzen dort, wo die Mitglieder der Verbände die Kosten der Externalisierung selbst wieder tragen müssen. Dies ist regelmäßig durch die steigenden Lohnnebenkosten der Fall. Allerdings sind Nutzen und Kosten von Vorruhestandsregelungen ungleich verteilt. Der Nutzen für einzelne Unternehmen übersteigt durchaus die anfallenden Kosten steigender Lohnnebenkosten. Dies gilt insbesondere dann, wenn die Unternehmen groß und bürokratisch organisiert waren und zugleich einem hohen Rationalisierungsdruck ausgesetzt waren, wie viele der großen Unternehmen der verarbeitenden Industrie der Achtzigerjahre. Diese Unternehmen hatten zudem ein größeres Problem mit ihrer alternden Belegschaft. Zudem spielt hier auch der Zeithorizont eine wesentliche Rolle: Während der positive Effekt der Vorruhestandsregelung sofort im Unternehmen zu spüren ist, so stellen sich die Kosten höherer Lohnnebenkosten erst langsam und mit einer beträchtlichen Verzögerung ein. Man kann auch unterstellen, dass zu Beginn der Vorruhestandsregelung keiner der Akteure voraussehen konnte, in welchem Umfang diese tatsächlich genutzt werden würden. Der Effekt der Externalisierung wurde so auch erst dann sichtbar, als das Instrument von vielen in Anspruch genommen wurde. 
Arbeitnehmer. Für die Tarifparteien stellt die rechtliche Mindestabsicherung der Arbeitnehmer die Machtposition des jeweiligen Verbandes in Tarifverhandlungen dar. Änderungen im Arbeitsrecht wie auch in den Leistungen der Systeme sozialer Sicherung haben direkte Implikationen für die Tarifparteien, in dem sie entweder die eine oder andere Tarifpartei direkt beeinflussen - wie etwa in Deutschland bei der Änderung des Paragraph 116 Arbeitsförderungsgesetz 1984, der Leistungen für vom Streik betroffene Arbeitnehmer vorsah - oder Tarifverhandlungen mit neuen Gegenständen konfrontieren - wie bei der Abschaffung der Lohnfortzahlung im Krankheitsfall im Jahr 1996. Eine Rücknahme arbeitsrechtlicher Standards beeinflusst daher die Verhandlungsposition der Gewerkschaften negativ, während ein Ausbau derselben ihre Position positiv berührt. Für die Arbeitgeber gilt dies umgekehrt. Mit der Androhung der einseitigen Benachteiligung einer Seite kann der Staat ein Drohpotenzial aufbauen und so eine widerspenstige Seite der Tarifparteien an den Verhandlungstisch zwingen.

Bei der Verhandlung sozialer Pakte nutzen Regierungen ihre Möglichkeit, in das Verhältnis zwischen den Tarifparteien zu intervenieren, um durch die (Wieder-)Herstellung eines hierarchischen Verhandlungssystems die Gemeinwohlorientierung der verhandelten Entscheidungen zu stärken. Der ,Schatten der Hierarchie' (Scharpf 1993: 145) dient damit nicht nur Wiedererlangung der Federführung der Politik in bereits verselbstständigten selbstkoordinierten Verhandlungssystemen, sondern auch der Repräsentation bestimmter gemeinwohlorientierter Positionen. Dabei ist die Herstellung von Hierarchie durch Regierungshandeln entscheidend, nicht jedoch notwendigerweise die Intervention oder der massive Druck auf die Tarifparteien an sich, wie die folgenden Beispiele zeigen. ${ }^{8}$

In einigen Ländern entstanden soziale Pakte erst durch die Drohung der Intervention durch den Staat. In den Niederlanden ließ die Regierung keinen Zweifel daran, dass sie in das Tarifsystem eingreifen würden, wenn sich die Tarifparteien nicht bewegen (Visser/Hemerijck 1997: 147). Erst unter einer massiven Drohung der Regierung Lubbers bewegten sich Gewerkschaften und Arbeitgeberverbände, die in den späten siebziger und frühen Achtzigerjahren Erfahrungen mit Regierungsinterventionen in die Tarifpolitik gemacht hatten, aufeinander zu. Im Schatten einer Interventionsdrohung waren sie selbst - ohne die moderierende Rolle der Regierung - in der Lage das Abkommen von Wassenaar auszuhandeln.

Entscheidend war dabei auch der hohe Druck, unter dem sich die Regierungen befanden. In den Niederlanden erwuchs der hohe politische Druck aus dem Scheitern der großen Koalition, die nach den Wahlen 1981 gebildet worden war und bereits nach einem Jahr durch die Mitte-Rechts Koalition unter Lubbers ersetzt wurde. In Italien fiel die Vereinbarung zur Abschaffung der Lohnindexierung mit dem Kollaps des politischen Systems zusammen, dem mehrere so genannte ,technokratische' Regierungen (Ciampi, Dini) folgten, die an politischer Legitimation durch Gewerkschaften und Arbeitgeberverbänden suchten. Unter politischen und ökonomischen Krisenbedingungen lassen sich Legitimationsverluste der Verbände leichter für Konzertierungen mobilisieren.

Der belgische Fall zeigt jedoch, dass die Herstellung von Hierarchie durch die Interventionsdrohung der Regierung keine hinreichende Bedingung für den erfolgreichen Abschluss von sozialen Pakten ist. In Belgien hatte die Interventionsdrohung der Regierung den gegenteiligen Effekt. Der wiederholte Eingriff der belgischen Regierung in die Tarifpolitik hat

8 Regini (1999), van Wijnbergen (1999) und Visser (1998) schreiben der Interventionsdrohung der Regierung die Rolle einer notwendigen Bedingung zu. 
dazu geführt, dass die Verbände sich aus ihrer Regulierungsfunktion eher zurückzogen und nicht bereit waren, die Verantwortung für dreiseitige Vereinbarungen zu übernehmen. Sie gingen berechtigterweise davon aus, dass die Regierung ihre Vorstellungen auch ohne die Mitarbeit der Verbände durchsetzen würde. Nach dem Scheitern der Paktverhandlungen hat die Regierung ein Gesetz zur „Förderung von Beschäftigung und Sicherung der Wettbewerbsfähigkeit" tatsächlich umgesetzt. Dieses Gesetz enthält die Regulierungen, gegen die sich die Gewerkschaften zuvor vehement gewehrt hatten, nämlich eine Koppelung belgischer Lohnerhöhungen an die Lohnentwicklung der drei wichtigsten Handelspartner (Vilrokx 1998). Nach der gesetzlichen Intervention in die Tarifpolitik konnten die Tarifparteien anschließend nur noch einen intersektoralen Tarifvertrag abschließen, der ihnen von der Regierung weitgehend vorgegeben war. Wenn hierarchisches Regierungshandeln die Mechanismen der Selbstkoordinierung regelmäßig außer Kraft setzt, dann können die korporativen Akteure von der Regulierung der Regierung profitieren, ohne die Verantwortung übernehmen zu müssen. Für sie gibt es keinen Anreiz zur Änderung ihrer nicht-kooperativen Haltung.

Die Interventionsmöglichkeit der Regierung ist zudem auch keine notwendige Bedingung, wenn die Verbände die Gefahr ihrer Marginalisierung antizipieren und bereits frühzeitig Gegenstrategien entwickeln. Dies kann man in Irland und Portugal beobachten: Hier ging die Initiative zur Vereinbarung eines Beschäftigungspakts von den Gewerkschaften aus, die eigenständig versuchten, auf die nationale Wirtschafts- und Sozialpolitik Einfluss zu nehmen. Der irische Gewerkschaftsbund ICTU bot der Regierung Mitte der Achtzigerjahre die Verhandlung eines 10-Jahresplans an (Aust 1999). Ein gemeinsamer Strategiebericht des Nationalen Wirtschafts- und Sozialrat (NESC) über die tiefe Krise der irischen Wirtschaft führte Mitte der Achtzigerjahre zu einer Initiative der irischen Gewerkschaften, die der Regierung ihren eigenen Plan zur Rettung der irischen Volkswirtschaft vorlegten (Visser 1998). Dieser Vorschlag wurde zunächst von der sozialliberalen Regierung abgelehnt und erst der Regierungswechsel zu Fianna Fail im Jahr 1987 führte zu dem angestrebten Bündnis. Die Regierung unter Charles Haughey suchte zunächst den Konsens mit den Gewerkschaften, den sie dann den Arbeitgebern und den Bauernverbänden geradezu aufzwang. In ähnlicher Weise haben in Portugal die Gewerkschaften UGT und CGTP bereits in den frühen Neunzigerjahren eigene Pläne mit Vorschlägen zur ,Modernisierung Portugals' vorgelegt, die später die Grundlage für den portugiesischen Beschäftigungspakt bildeten. In diesen Fällen haben die Arbeitnehmerorganisationen den Regierungen explizit angetragen, dreiseitige Verhandlungen aufzunehmen, um die staatliche Steuerung bei der Überwindung der Beschäftigungskrise zu verstärken und die glaubhafte Alternative einer drohenden politischen Marginalisierung der Verbände abzuwenden.

\section{Wie Gewerkschaften ihre neue Position in der Beschäftigungspolitik verarbeiten}

Grundsätzlich kann man nicht davon ausgehen, dass die Lösung des Beschäftigungsproblems Bestandteil der Interessenpolitik der Tarifparteien ist, zumindest nicht insoweit wie die vorgeschlagenen Instrumente ihren traditionellen Interessendefinitionen widersprechen. Die Gewerkschaften haben in der Frage der Bekämpfung von Arbeitslosigkeit traditionell nur Lösungsinstrumente unterstützt, die sowieso den Interessen ihrer Kernmitglied- 
schaft entsprachen: kollektive Arbeitszeitverkürzungen und eine nachfrageorientierte Wirtschaftspolitik, die mit Lohnsteigerungen einher ging. Die Gewerkschaften sind die potenziellen Verlierer der Reformpolitik sozialer Pakte: „Reducing the rate of growth of real wages, weakening employment protection, or cutting sickness and disability benefits are to the disadvantage of the insiders on the labor market (the majority of union members) with the stated aim of improving the chances of those (usually non-unionized) at the fringes of the labor market" (van Wijnbergen 1999: 12).

Allerdings entwickeln die Tarifparteien dann ein größeres Eigeninteresse an der Lösung des Beschäftigungsproblems, wenn die Nichtbehandlung des Problems den eigenen Verband in Frage zu stellen droht. Dies kann auf mehreren Weisen geschehen: zum Beispiel dadurch, dass die hohe Arbeitslosigkeit die Tarifpolitik erschwert, in dem sie die Machtpositionen verschiebt. Für Arbeitgeberverbände kann dies auch zum Problem werden, wenn Unternehmen zunehmend ihre eigene Tarifpolitik gegen die Interessen des Verbands betreiben. Arbeitslosigkeit kann auch dann ein Problem für die Verbände werden, wenn die gesellschaftliche Legitimation der Tarifpolitik sinkt, z.B. mit der zunehmenden Kritik an Arbeitsmarktkartellen und Insider-Outsider Problemen. Aber auch wenn die bestehenden Blockaden von ihnen selbst als hinderlich erkannt werden, sind die traditionellen Positionen, die sich auf die Befriedigung der Kernmitgliedschaft stützt, in der Regel die anerkannten und damit kurzfristig sinnvolleren.

Unter welchen Voraussetzungen wechseln Verbände ihre Strategien? Gewerkschaften werden dann ihre Position wechseln, wenn sie befürchten müssen, ansonsten politisch marginalisiert zu werden. Diese Gefahr der Marginalisierung kann mit Mitgliederschwäche einhergehen, muss jedoch nicht. Es lässt sich kein direkter Zusammenhang zwischen Mitgliederverlusten der Gewerkschaften und sozialen Pakten herstellen. Die Drohung der Marginalisierung kann auch von der Interventionsdrohung der Regierung ausgehen. Allerdings genügt die entschlossene Haltung der Regierung nicht, wie oben deutlich wurde. Wenn die Interventionsdrohung der Regierung von den Gewerkschaften nicht als ein empfindliches Übel empfunden wird, brauchen sie sich auf Paktverhandlungen nicht einzulassen. So lange Gewerkschaften davon ausgehen können, über ihre Funktionen in der Tarifpolitik und betrieblichen Interessenvertretung auch langfristig in ihrer Organisation stabil bleiben zu können, können die in einem sozialen Pakt angelegten Nachteile die Vorteile überwiegen und die Gewerkschaft wird sich nicht ernsthaft an einem solchen Tausch beteiligen. Der deutsche Fall zeigt zudem, dass soziale Pakte besonders schwer zu verhandeln sind, wenn erstens die Regierung nur wenig Drohpotenzial besitzt und zweitens die Verbände sich auf Grund ihrer starken institutionellen Verankerung weitgehend von dem Druck des Arbeitsmarktes isolieren können (Ebbinghaus und Hassel 2000).

Wenn sich Gewerkschaften auf soziale Pakte einlassen, dann lassen sich die klassischen innerorganisatorischen Verarbeitungsmechanismen von Verbänden beobachten, die im Zuge der Stärkung ihrer politischen Kompromissfähigkeit Mitgliederinteressen neu interpretiert: ${ }^{9}$ Im politischen Tausch bringt die Gewerkschaftsseite ihre Zustimmung zu politi-

9 Das folgende Argument basiert auf den Ausführungen von Alessandro Pizzorno über den politischen Tausch und kollektive Identitäten. Pizzorno nutzt den Zusammenhang, um zu erklären, unter welchen Bedingungen Gewerkschaften bestehende Formen des politischen Tauschs auf Grund veränderter kollektiver Identitäten nicht mehr aufrecht erhalten können. Meines Erachtens lassen sich diese Beobachtungen auf die Entstehungsbedingungen eines neuen politi- 
schen Reformvorhaben ein. Sie muss dabei im Interesse ihrer Kompromissfähigkeit in politischen Verhandlungen von den unmittelbaren Mitgliederinteressen an Lohnzuwächsen und sozialer Sicherung abstrahieren. Daraus folgen zwei wesentliche strategische Optionen: Erstens kann eine Gewerkschaft im politischen Tausch eher als im reinen Tarifgeschäft, langfristige Ziele auf Kosten von kurzfristigen verfolgen. Zweitens erhält sie im politischen Tausch eine politische Gestaltungsmöglichkeit im Unterschied zu einer direkten Bedürfnisbefriedigung ihrer Mitglieder. ${ }^{10}$ Die Unterscheidung zwischen kurz- und langfristigen Zielen wie auch zwischen direkten Mitgliederbedürfnissen und politischer Gestaltungsmöglichkeit sind symmetrisch verteilt. Je mehr sich die Organisation in ein politisches Geschäft mit der Regierung begibt, desto langfristiger wird ihre Zielsetzung sein wird (wie z.B. die Lohnzurückhaltung). Verfügt sie dazu noch über eine relatives Interessenvertretungsmonopol, so wächst ihre Fähigkeit die möglichen Interessen ihrer Mitglieder in ihrem - langfristig strategischen - Interesse zu formulieren und zu re-interpretieren (Pizzorno 1978: 279ff.). Vor diesem Hintergrund basiert der in sozialen Pakten angelegte politische Tausch darauf, dass die Gewerkschaftsseite die Interessen ihrer Mitgliedschaft neu definiert, um eine kurzfristige bedürfnisorientierte Interessenpolitik in eine langfristige organisationsorientierte Interessenpolitik zu übertragen. Dabei können Gewerkschaften auf bestehende Interessendivergenzen innerhalb ihrer Mitgliedschaft zurückgreifen.

Auch innerhalb der Gewerkschaften sind die Gruppen der Nutznießer und Kostenträger von Regulierungen des Sozialstaates und des Arbeitsmarktes nicht deckungsgleich. Zum Beispiel ist eine prinzipielle Konfliktlinie innerhalb der Verbände das Verhältnis von Sozialund Tarifpolitik im Hinblick auf die Bereitstellung und Finanzierung sozialstaatlicher Dienstleistungen. Ein beträchtlicher Bestandteil sozialstaatlicher Leistungen resultiert in Beschäftigungsverhältnissen in der Sozialverwaltung und im Bereich sozialer Dienstleistungen. Die Sozialverwaltung wie auch soziale Dienstleistungen sind ein zunehmend großer und wachsender Bestandteil öffentlich finanzierter Beschäftigung und damit sowohl Teil der Lösung wie auch ein Teil des Problems. Als Teil der Lösung bieten sie Beschäftigungsmöglichkeiten im Dienstleistungssektor, als Teil des Problems stellen sie einen nicht unbeträchtlichen Kostenfaktor dar. Die Interessenlagen von Gewerkschaften sind daher im Bezug auf soziale Dienstleistungen gespalten: Je nach Fachzuständigkeit innerhalb der Gewerkschaften gilt Beschäftigung in sozialen Dienstleistungen überwiegend als Kostenfaktor oder als Beschäftigungschance. Auf der einen Seite müssen Gewerkschaften die Beschäftigteninteressen der öffentlich Beschäftigten als wachsende Mitgliedergruppe im Blick haben. Sie müssen sich zumindest für den Status Quo in der Bereitstellung von sozialen Dienstleistungen einsetzen. Auf der anderen Seite werden auch Gewerkschaften durch die zunehmenden finanziellen Belastungen von Lohnnebenkosten beeinträchtigt. Insbesondere in wettbe-

schen Tauschs übertragen (Pizzorno 1978). Siehe ganz analog dazu die Rolle der Verbände als Private Interest Governments bei Streeck und Schmitter (Streeck und Schmitter 1985).

10 Im Unterschied zu Pizzorno könnte man auch annehmen, dass es im langfristigen Interesse der Arbeitnehmer selbst ist, an einem politischen Tausch teilzunehmen, selbst wenn dadurch kurzfristige Lohninteressen nicht direkt befriedigt werden. Voraussetzung dafür ist, dass die Regierung den Interessen der Gewerkschaften entgegen kommt: zum einen als ,Kompensator', indem sie Gewerkschaften für ihre Opfer entschädigt. Zum anderen als ,Garant', indem sie sicher stellt, dass die Beschäftigten an der zukünftigen wirtschaftlichen Entwicklung auch partizipieren (Lange 1984; Alvarez, Garrett, Lange 1991). 
werbsorientierten Sektoren müssen auch Gewerkschaften prinzipiell ein Interesse an der Reduzierung von Kosten im sozialen Dienstleistungssektor haben.

Neue Konfliktlinien zwischen geschützten Sektoren im öffentlichen Dienst und wettbewerbsorientierten Sektoren zeigen sich am deutlichsten in Ländern mit einem ausgedehnten Beschäftigungsanteil in sozialen Dienstleistungen. Hier finden sich Interessenkonflikte innerhalb der Gewerkschaften, die quer zu traditionellen Interessenpositionen liegen. Die zunehmende Zentralisierung der Tarifpolitik in Schweden in den Siebzigerjahren wurde zum Beispiel darauf zurückführt, dass beide Tarifparteien in den wettbewerbsorientierten Sektoren versuchten, die Ausgaben für den öffentlichen Sektor zu kontrollieren (Swenson 1992; Clayton und Pontusson 1998). Man kann daher grundsätzlich nicht erwarten, dass Tarifparteien einheitliche Positionen im Bezug auf die Finanzierung von sozialen Dienstleistungen verfolgen, sondern dass es innerhalb der Verbände konfligierende Interessen in Bezug auf die Reform bestehender Sozial- und Tarifsysteme gibt.

Die durch die Federführung der Regierung wiederhergestellte Zentralisierung der Entscheidungsfindung ermöglicht zudem solche Neupositionierungen, da hier die Verbände zu einer kohärenten Position finden müssen. Oftmals sind unterschiedliche Vertreter der gleichen Organisationen in den Selbstverwaltungsgremien der Arbeits- und Sozialverwaltung und in der Tarifpolitik vertreten. Auf Grund der funktionellen Zuordnung dieser Vertreter zu den entsprechenden Politikbereichen (Arbeitsmarkt-, Sozial- und Tarifpolitik) entwickeln sie für dieses Politikfeld spezifische Positionen, die regelmäßig auch klassenübergreifend angelegt sind. Diese können in einem latenten Widerspruchsverhältnis zu den Positionen ihres Verbands in anderen Politikfeldern stehen. Die Überschneidung von Akteuren der gleichen Verbände in verschiedenen Verhandlungssystemen eröffnet den politischen Akteuren innerhalb zentralisierter Formen der Konzertierung die Möglichkeit, Verbändeinteressen und -positionen einander gegenüberzustellen und über widersprüchliche Konfliktlinien zwischen Verbänden und innerhalb der Verbände neue gemeinsame Handlungsspielräume zu schaffen. Durch die Rückverlagerung einzelner sozial- und tarifpolitischer Themen auf die Ebene der Spitzenvertreter der Verbände müssen verbandsinterne Meinungsverschiedenheiten gelöst und neue Positionen geschaffen werden.

Empirisch betrachtet wird deutlich, dass solche Neupositionierungen nach einer Interventionsdrohung der Regierung abrupt erfolgen und scheinbar nicht langfristig vorbereitet werden. In den Niederlanden verfolgten die Gewerkschaften in den Jahren vor Wassenaar (1982) eine konfliktreiche Strategie der Arbeitszeitverkürzung, die sich an die Vorgaben des Europäischen Gewerkschaftsbunds anlehnten. Dies wurde von Arbeitgebern als Element zur Lösung arbeitsmarktpolitischer Probleme kategorisch abgelehnt. In Italien fand kurz vor dem Abkommen von 1992 noch ein Generalstreik der Gewerkschaften gegen die Abschaffung der Lohnindexierung statt. Am Tag nach der Unterzeichnung der Vereinbarung bot Bruno Trentin, der damalige Vorsitzende der CGIL, seiner Organisation den Rücktritt an. Diese rapiden Positionswechsel lassen vermuten, dass Gewerkschaften über ein flexibles Repertoire an Rhetorik über beschäftigungspolitische Zusammenhänge verfügen, das auch kurzfristig mobilisiert werden kann. ${ }^{11}$

11 Natürlich wissen auch Gewerkschaftsführer, dass zwischen Löhnen und Beschäftigungsmöglichkeiten ein Trade-Off besteht, über den sie im wesentlichen in Tarifverhandlungen bestimmen. Auch die deutschen Gewerkschaften konnten die einzelnen Bestandteile wahlweise rhetorisch einsetzen, indem sie zunächst bei der Zwickel-Initiative von 1995 Lohnzurückhaltung im 


\section{Gemeinwohlorientierung und die Instrumentalisierung von Kooperation}

Eine Vereinbarung zwischen Regierung und Verbänden muss nicht automatisch gemeinwohlorientiert sein. ${ }^{12}$ Regierungen können Vereinbarungen schließen, um ihre eigene Legitimation zu verbessern, ohne mit dieser Vereinbarung der Problemlösung näher zu kommen. Verbände können Vereinbarungen mit der Regierung nutzen, um den Interessen ihrer Mitglieder zu dienen, ohne dass man der Problemlösung näher kommt. So es lassen sich Szenarien entwickeln, in denen Regierung und Tarifparteien Lösungen vereinbaren, deren Effekt eine zeitweilige Reduzierung der Arbeitslosenzahlen ist, die das Beschäftigungsproblem jedoch langfristig verstärken (Berthold/Hank 1999). ${ }^{13}$ Daher stellt sich die Frage, wie die Gemeinwohlorientierung solcher Vereinbarungen gesichert werden kann.

Hier lassen sich zwei Bedingungen zur Wahrung von Gemeinwohlinteressen feststellen. Erstens gilt auch hier, dass die Zentralisierung der Entscheidungsfindung dazu beiträgt, dass negative Externalitäten einzelner Entscheidungen von allen Beteiligten internalisiert werden. Da in den Vereinbarungen Tauschgeschäfte zwischen den wesentlichen Akteuren auf dem Arbeitsmarkt über die Behandlung des Beschäftigungsproblems verabredet werden, kann man davon ausgehen, dass die Umsetzung dieser Entscheidungen alle Gruppen in einem ähnlichen Maße trifft. Dafür ist jedoch zweitens die Beteiligung aller wesentlichen Tarifparteien an den Vereinbarungen notwendig. Nur durch die gleichberechtigte Beteiligung aller wesentlichen Akteure lässt sich verhindern, dass Vereinbarungen einseitig zu Lasten von entweder Steuerzahlern, Arbeitnehmern oder Unternehmen durchgesetzt werden können.

Damit ist die Gefahr der Instrumentalisierung sozialer Pakte für einseitige verbandliche Interessen dort am größten, wo entweder nicht alle Gruppen durch Verbände vertreten werden oder wichtige Arbeitsmarktakteure nicht am Verhandlungstisch sitzen. Im ersten Fall können dies entweder nicht organisierbare Interessen sein (zukünftige Generation) oder aber Interessen betreffen, die sich nicht in der Mitgliedschaft der Verbände widerspiegeln (z.B. junge Arbeitnehmer in den Gewerkschaften). In beiden Varianten steigt die Anforderung an die Regierung, als Fürsprecherin für die schlecht organisationsfähigen allgemeinen Interessen aufzutreten (Mayntz 1992).

Im zweiten Fall sind dreiseitige Vereinbarungen von Beginn an, und auch im weiteren Verlauf, größeren Risiken des Scheiterns ausgesetzt. Zum Beispiel haben in Portugal zwar beide großen Gewerkschaften, die CGTP und die UGT, ihre eigenen Vorschläge zur Modernisierung Portugals vorgelegt. Der Strategische Pakt wurde 1997 jedoch nur zwischen

Tausch mit Beschäftigungszusagen anboten, drei Jahre später deutliche Lohnzuwächse mit der mangelnden Binnennachfrage begründeten. Im Rahmen der Diskussion über die ,Rente ab 60' schwenkt nun die Gewerkschaftsführung wieder auf die Linie der langfristigen Lohnzurückhaltung im Interesse der Beschäftigungsförderung ein. Im Interesse der Mobilisierung ihrer Mitgliedschaft im Verlauf von Lohnverhandlungen ist es allerdings für die Gewerkschaftsseite wesentlich, daß Lohninteressen nicht im Widerspruch zu Beschäftigungsinteressen erscheinen.

12 Unter Gemeinwohlorientierung sei mit dem ,Kaldor-Kritierum' die Verfolgung derjenigen Maßnahmen zur Problemlösung verstanden, in denen der Nutzen der Begünstigten die Kosten der Benachteiligten übersteigt (Scharpf 1991b: 15). Da es sich um objektiv sinnvolle Maßnahmen im Hinblick auf das zu lösende Problem handeln muss, kann damit auch die Auswahl möglicher Reformen und Instrumente einer Gemeinwohlprüfung unterzogen werden.

13 Siehe die Möglichkeiten der Externalisierung von Rationalisierungskosten der Tarifparteien in Fußnote 7. 
der Regierung, den Arbeitgebern und der kleineren, aber regierungsnäheren Gewerkschaft UGT abgeschlossen, die nur etwa ein Viertel der portugiesischen Gewerkschaftsmitglieder vertritt. Die ehemals kommunistische Gewerkschaft CGTP hat sowohl den Pakt als auch seine Umsetzung boykottiert. Sie hat weder das Abkommen von 1990 noch das von 1996 unterschrieben. Sie ist daher auch nicht Mitglied des Überwachungsausschuss.

Damit fehlt der Vereinbarung von Beginn an die notwendige Unterstützung der Mehrheitsfraktion der Gewerkschaften. Die sozialistische Regierung hat zudem versucht, die traditionell ihr nahe stehende UGT zu stützen. ${ }^{14}$ Die intergewerkschaftliche Rivalität belastet den Sozialpakt auch dadurch, dass sich die CGTP nicht an die vereinbarten Leitlinien halten muss und sich daher auf Kosten der in dem Pakt festgeschriebenen Reformelemente profilieren kann (Barreto und Naumann 1998: 421). Da dies zumeist auf Kosten der Arbeitgeberseite erfolgt, ist das Interesse der Arbeitgeber an dem Strategischen Pakt wieder deutlich abgekühlt. Dies hat wiederum Rückwirkungen auf die Ernsthaftigkeit der Regierung bei der Umsetzung ihrer Reformversprechen.

Im Fall Portugals basiert der Sozialpakt daher eher auf einer Allianz zwischen der sozialistischen Regierung und der ihr nahe stehenden Gewerkschaft. Beide Seiten nutzten den Pakt dafür ihre eigene Legitimation zu stärken. Die UGT konnte ihre tatsächliche Schwäche in den einzelnen Branchen durch ein konzertiertes Vorgehen überspielen, während die Regierung von den Tarifparteien unterstützte Reformvorhaben in den Pakt aufnahm. Die Arbeitgeber unterstützten den Pakt, um ihre Position gegenüber der radikaleren CGTP zu stärken. Die gut 300 Einzelmaßnahmen umfassende Vereinbarung ist dabei für alle Beteiligten in jeder Hinsicht benutzbar, dient jedoch kaum der Verabredung einer ernsthaften Reformstrategie auf dem Arbeitsmarkt. Die im Pakt vorgesehene Umsetzungskommission ist faktisch nicht arbeitsfähig. Gleichzeitig hat die Regierung begonnen direkte Gespräche mit den Tarifparteien zu führen, um wieder in Kontakt mit der CGTP zu kommen.

Die Bedeutung eines gemeinsamen Vorgehens der Tarifparteien für die Etablierung von Kooperationsbeziehungen zeigt sich auch am Kontrast des portugiesischen Falls mit der Situation in Italien. Die Bemühungen um die Einheit der drei großen italienischen Gewerkschaftsbünde in den Neunzigerjahren sind der Erkenntnis geschuldet, dass eine größere Handlungsfähigkeit der Gewerkschaften innerhalb tripartistischer Absprachen nur durch eine größere Zusammenarbeit zwischen den Gewerkschaftsbünden erreichbar ist. $^{15}$

14 Die UGT war mit über 3 Millionen US \$ verschuldet, die sie mit Hilfe einer Regierungsbürgschaft bei einer staatlichen Bank umschulden konnte. Diese indirekte Unterstützung war jedoch insofern kontraproduktiv, als dass die zeitliche Nähe zwischen der Umschuldung und dem Abschluss des Strategischen Pakts von der Öffentlichkeit als politische Vorteilsnahme interpretiert wurde. Der Strategische Pakt war daher politisch bereits diskreditiert, bevor er begann. Eine besonders unangenehme Komponente der Transaktion für die Regierung war die weit bekannte Tatsache, dass sich die finanziellen Probleme der UGT auf Unregelmäßigkeit und Korruptionsvorwürfe zurückgeführt werden müssen, die dazu geführt hatten, dass die EU-Kommission alle weiteren Zahlungen für Aktivitäten der UGT im Bereich der beruflichen Bildung eingefroren hatte (Barreto und Naumann 1998).

15 Ähnliche Kooperationsbestrebungen zwischen den traditionell verfeindeten Gewerkschaftsbünden CCOO und UGT kann man auch bei den spanischen Gewerkschaften im Kontext der Paktbemühungen seit 1994 beobachten (Perez 1999: 26). 


\section{Die Folgen: Wiedererlangung nationaler Handlungsfähigkeit}

Allerdings haben die beteiligten Parteien auch nach Abschluss dreiseitiger Vereinbarungen noch immer das Problem, dass ihr eigenes Interesse an der Lösung des Beschäftigungsproblems von der anderen Seite für ihre Zwecke missbraucht werden kann. Z.B. kann Lohnzurückhaltung der Gewerkschaften als Mittel der Beschäftigungspolitik von den Unternehmen in Anspruch genommen werden, ohne dass Beschäftigung wirklich entsteht. Die Akteure, insbesondere die Regierung und die Gewerkschaften, profitieren erst dann von dem Pakt, wenn er Resultate zeigt. Das anvisierte Beschäftigungswachstum durch institutionelle Reformen ist allerdings ein langfristiger Prozess, der kaum zu kurzfristigen Erfolgen führt. Zudem werden nicht alle Reformprojekte gleichzeitig angegangen und wesentliche Reformschritte kommen erst nach einigen Jahren auf den Verhandlungstisch, wenn die Geduld der einzelnen Verbände bereits erschöpft ist.

Die Asymmetrie zwischen den Vorleistungen der Gewerkschaften und der unbestimmten Hoffnung auf ein dadurch induziertes Beschäftigungswachstum führte dementsprechend zu mehr Konflikten und Unzufriedenheit auf der Gewerkschaftsseite als bei den anderen Teilnehmern sozialer Pakte. Auch nach dem Abschluss von Sozialen Pakten gab es Arbeitskämpfe und größere Proteste gegen weiterführende Reformen. Die Vereinbarungen führten somit nicht zu einem automatischen Einverständnis der Gewerkschaften gegenüber allen sozialpolitischen Reformvorhaben der Regierung. In den Niederlanden konnten z.B. die Kürzungen der Ansprüche der Erwerbsunfähigkeitsrente 1993 nur unter massivem Protest der Gewerkschaften und unter erneuter Interventionsdrohung der Regierung vorgenommen werden. In Italien hat sich die Stimmung der Tarifparteien 1996/97 im Vergleich zu den Jahren 1993 bis 1995 deutlich verschlechtert.

Aus diesen Gründen besteht insbesondere nach Ablauf einer Schonfrist von einigen Jahren das Problem, dass die Vereinbarungen zwischen den Beteiligten auf eine andere - insti-

Tabelle 2: Gemeinsame Institutionen der Tarifparteien

\begin{tabular}{|c|c|c|c|}
\hline Länder & Institutionen & Zusammensetzung & Funktion \\
\hline \multirow[t]{4}{*}{ Irland } & $\begin{array}{l}\text { National Economic and Social } \\
\text { Council }\end{array}$ & $\begin{array}{l}\text { Arbeitgeber, Gewerkschaften } \\
\text { und Regierung }\end{array}$ & Konsultation \\
\hline & $\begin{array}{l}\text { Employer-Labour } \\
\text { Conference (1970) }\end{array}$ & $\begin{array}{l}\text { Arbeitgeber, Gewerkschaften } \\
\text { und Regierung }\end{array}$ & Lohnverhandlungen \\
\hline & Labour Court & $\begin{array}{l}\text { Arbeitgeber und } \\
\text { Gewerkschaften }\end{array}$ & Schlichtung \\
\hline & Joint Labour Committees & $\begin{array}{l}\text { Arbeitgeber und } \\
\text { Gewerkschaften }\end{array}$ & $\begin{array}{l}\text { handeln } \\
\text { Mindestlöhne aus }\end{array}$ \\
\hline \multirow[t]{2}{*}{ Niederlande } & $\begin{array}{l}\text { Stichting van de Arbeid } \\
(1945)\end{array}$ & $\begin{array}{l}\text { Arbeitgeber und } \\
\text { Gewerkschaften }\end{array}$ & Beratungsorgan \\
\hline & $\begin{array}{l}\text { Sociaal-Ekonomische Raad } \\
(1950)\end{array}$ & $\begin{array}{l}\text { Arbeitgeber, Gewerkschaften } \\
\text { und Regierung }\end{array}$ & $\begin{array}{l}\text { Beratung, } \\
\text { Gutachter }\end{array}$ \\
\hline Italien & $\begin{array}{l}\text { Consiglio Nazionale dell' Eco- } \\
\text { nomia e del Lavoro (CNEL) } \\
\text { Nationaler Wirtschafts- und } \\
\text { Arbeitsrat }\end{array}$ & $\begin{array}{l}\text { Arbeitgeber, } \\
\text { Gewerkschaften und } \\
\text { Regierung }\end{array}$ & $\begin{array}{l}\text { Forschung, } \\
\text { Information, } \\
\text { Beratung }\end{array}$ \\
\hline
\end{tabular}


Tabelle 3: Durch Soziale Pakte geschaffene Institutionen

\begin{tabular}{llll}
\hline Länder & Institution & Zusammensetzung & Funktion \\
\hline Italien & $\begin{array}{l}\text { Nationalen Rat für Berufsbil- } \\
\text { dung (1996) }\end{array}$ & $\begin{array}{l}\text { Arbeitgeber, Gewerkschaften } \\
\text { und Regierung (mit Beteili- } \\
\text { gung der Regionen) }\end{array}$ & $\begin{array}{l}\text { Organisierung von eigenen } \\
\text { Ausbildungslehrgängen }\end{array}$ \\
Portugal & $\begin{array}{l}\text { Arbeitsschutzkommissionen } \\
\text { (1991) (nicht umgesetzt) }\end{array}$ & $\begin{array}{l}\text { Arbeitgeber und Gewerk- } \\
\text { schaften }\end{array}$ & $\begin{array}{l}\text { Verbesserung des Arbeits- } \\
\text { schutzes und der Partizipa- } \\
\text { tion der Arbeitnehmer im } \\
\text { Betrieb }\end{array}$ \\
& Überwachungsausschuss & $\begin{array}{l}\text { Regierung, Arbeitgeber und } \\
\text { Gewerkschaften } \\
\text { (ohne CGTP) }\end{array}$ & $\begin{array}{l}\text { Uberwachung des Strategi- } \\
\text { schen Sozialpakts }\end{array}$ \\
& & $\begin{array}{l}\text { Arbeitgeber und Gewerk- } \\
\text { schaften }\end{array}$ & Schlichtungsbehörde \\
& Labour Relations & Arbeitgeber und Gewerk- & Überwachung des PNR \\
& Commission (1991) & schaften & \\
\hline
\end{tabular}

tutionelle - Art stabilisiert werden müssen. In den meisten Ländern lassen sich institutionelle Stabilisierungen der Verbände auch direkt auf die Sozialpakte zurückführen. Eine wichtige Form der Stabilisierung sozialer Pakte bestand in der Revitalisierung bestehender tripartistischer Einrichtungen. Zunächst wurden schon durch den Abschluss der Vereinbarung die entsprechenden Gremien formal wieder aufgewertet. Sie wurden auch danach wieder besser gepflegt und die Konsultationen in diesen Gremien wurden wieder aufgenommen. In den Niederlanden war zwar die Stiftung für Arbeit ein traditionelles korporatistisches Gremium, das von den Tarifparteien in der unmittelbaren Nachkriegszeit gegründet worden war. In den Siebzigerjahren und bis in die frühen Achtzigerjahre hatte es jedoch ein eher bescheidenes Dasein gefristet. Und selbst nach Abschluss der Vereinbarung von Wassenaar bestanden in den späten Achtzigerjahren Überlegungen dieses Gremium abzuschaffen. Erst im Zuge der Diskussion des niederländischen Modells wurde die Stiftung für Arbeit und mit ihr die Konstruktion der sozialen Konzertierung wieder neu bewertet. Auch in Italien war der CNEL in der Zeit vor dem Ciampi-Protokoll weitgehend verkümmert. Erst danach wurde sowohl seine Rolle aufgewertet als auch neue tripartistische Gremien zur Verbesserung der beruflichen Bildung geschaffen. Der italienische Beschäftigungspakt von 1996 sah vor, dass ein Nationaler Rat für Berufsbildung unter der Leitung des Arbeitsministeriums die Organisation und Reform der beruflichen Bildung voran treiben soll (siehe Tabelle 3).

Neue gemeinsame Einrichtungen wurden auch zur Evaluierung der Sozialen Pakte geschaffen. Oder gemeinsame Einrichtungen wurden gegründet, um Funktionen auszuüben, die bei der Verhandlung der Pakte als institutionell unzureichend entwickelt festgestellt wurden. In Irland wurde das Central Review Committee zur Überwachung des ersten ,Programme for National Recovery' geschaffen. Bestehende Probleme bei der Moderation von Tarifkonflikten sollten durch die Schaffung der Labour Relations Commission behoben werden. In Portugal sah ein Abkommen aus dem Jahr 1991, an dem auch die CGTP beteiligt war, die Einrichtung von Arbeitsschutzkommissionen vor. Auf Grund von Rivalitäten zwischen den Gewerkschaften wurde dieses Abkommen jedoch nicht umgesetzt.

Im italienischen Fall bestand ein institutionell unzureichend entwickelter Bereich in der betrieblichen Interessenvertretung durch die großen Dachverbände. Durch die Dezentrali- 
sierung der Tarifverhandlungen wurden einerseits betriebliche Verhandlungspartner erforderlich, andererseits war die Stellung der großen Gewerkschaften in den Betrieben prekär. Die großen italienischen Gewerkschaftsverbände litten bereits seit den frühen Achtzigerjahren darunter, dass sie zwar auf der politischen und betrieblichen Ebene über ein Vertretungsmonopol verfügten, tatsächlich jedoch die betriebliche Verankerung sehr schwach war und sie zunehmend der Konkurrenz von Berufsverbänden ausgesetzt waren (Regalia und Regini 1998). Daher war die Neuregulierung der betrieblichen Interessenvertretung ein direkter Bestandteil des Abkommens über Arbeitskosten vom 23. Juli 1993, das in einer gesonderten Vereinbarung über die Einheitlichen Gewerkschaftsvertretungen (RSU) im Dezember 1993 konkretisiert wurde (Telljohann 1998: 654). Die in der Vereinbarung geschaffenen Nachfolgerinstitutionen der früheren Fabrikräte sollten daher die Verbindung der Gewerkschaften in die Betriebe fördern. Um nicht weiteren Widerstand gegen das Vertretungsmonopol der großen Dachverbände zu provozieren, stellen die großen Gewerkschaften nur ein Drittel der Mitglieder, während zwei Drittel von der gesamten Belegschaft über Listen gewählt werden.

Infolge der erneuten Einbindung der Verbände in tripartistische Gremien etwa zur Förderung der beruflichen Bildung bieten sich den Verbänden auch Möglichkeiten der Legitimation gegenüber ihren Mitgliedern, die sie ansonsten eventuell in der Tarifpolitik oder im Widerstand gegen andere sozialpolitische Reformen gesucht hätten. Da tripartistische Gremien auch Ressourcen sowie Einflussmöglichkeiten auf die staatliche Politik bieten, werden die Verbände von der Verfolgung direkter Mitgliederinteressen entlastet. Zum Beispiel bietet die Reform der beruflichen Bildung durch gemeinsame Einrichtungen mit den Gewerkschaften im Fall Italiens auch die Möglichkeit für die Gewerkschaften, ihrer klientelistische Politik der älteren Arbeitnehmer in gesicherten Beschäftigungsverhältnissen - deren Ursprung in der extremen Überalterung der Gewerkschaften mit einem Rentneranteil von mehr als einem Drittel der Gesamtmitgliedschaft zu suchen ist - gegenzusteuern.

Diese institutionelle Neuordnung der Zuständigkeiten und Verfahren in der Abstimmung der Beschäftigungs- und Sozialpolitik hat insgesamt eine stabilisierende und handlungsbefähigende Wirkung auf die Verbände. Sie birgt natürlich auch die Möglichkeit neuer institutioneller Blockaden in sich, deren Wirkungsweise sich erst in der Zukunft erweisen wird. Für die Gegenwart scheint sie jedoch in jedem Fall die Handlungsfähigkeit der Verbände zu stärken, ohne dass dies notwendigerweise auf eine reine Konservierung der bestehenden Tarifsysteme der Nachkriegszeit hinauslaufen muss.

\section{Schluss}

Dreiseitige Vereinbarungen zwischen Regierungen und Tarifparteien zur Wiederbelebung der Arbeitsmärkte haben in den letzten Jahren in einer Reihe von europäischen Ländern an Bedeutung zugenommen. Sie wurden als Reaktion nationaler Regierungen auf zwei Entwicklungen ins Leben gerufen: auf den zunehmenden Wettbewerbsdruck im Rahmen der nahenden europäischen Wirtschafts- und Währungsunion und auf die Belastung der sozialen Sicherungssysteme durch anhaltende und steigende strukturelle Unterbeschäftigung. Innerhalb eines dreiseitigen Beschäftigungspakts wurden Kompensationsgeschäfte zwischen der Steuer- und Abgabenentlastung der Arbeitsmarktparteien, Beschäftigungspro- 
grammen durch die öffentliche Hand auf der einen Seite und Lohnzurückhaltung durch die Gewerkschaften auf der anderen Seite möglich.

Der Erfolg der Verhandlung sozialer Pakte ist abhängig von der Entschlossenheit der Regierung, institutionelle Reformen in der Arbeitsmarktregulierung durchzuführen sowie von der Empfindsamkeit der Verbände gegenüber den negativen Effekten der Beschäftigungskrise. Die dafür notwendige Strategiefähigkeit von Regierungshandeln ist insbesondere im Hinblick auf die Lohnpolitik bedeutsam. Eine moderate Lohnpolitik stand in allen Fällen am Beginn von Konzertierungsverhandlungen. In Ländern mit traditionell einflussreichen Gewerkschaften wurde diese Konzession bis zur Unterzeichnung der Sozialpakte von den Gewerkschaften abgelehnt. Nur in Ländern, in denen die Gewerkschaften noch weiteren Legitimationsverlusten ausgesetzt waren (wie durch die intergewerkschaftliche Konkurrenz im Fall Portugals und dem drohenden Vorbild Großbritanniens im irischen Fall), waren die Verbände bereit, selbst zum Abschluss dreiseitiger Vereinbarungen beizutragen. In den anderen Fällen waren beide Tarifparteien erst unter dem Druck der Regierungsintervention der sich im Übrigen auch gegen die Arbeitgeberseite richtete - in der Lage, die Modalitäten einer für beide Seiten erträglichen Lohnpolitik zu verhandeln.

Aus den Beispielen der nationalen Beschäftigungspakte lassen sich meines Erachtens zwei wesentliche Erkenntnisse ziehen: Erstens zeigen sie deutlich, dass eine politisch gesteuerte Re-integration bereits ausdifferenzierter Politikfelder mit verschiedenen Akteurskonstellationen in nationalen Verhandlungssystemen möglich ist. Nachdem die Steuerungstheorie von einer säkularen Tendenz der Verselbstständigung von Politikfeldern ausging, die zu einem zunehmenden Verlust der Handlungsfähigkeit des Staates führen müsse, findet man nun in der politischen Praxis Anzeichen für Eingriffe der Regierung in selbstkoordinierte Verhandlungssysteme der Tarifparteien. Während eines der wesentlichen Motive für konzertierte Verhandlungen über Fragen der Arbeitsmarkt- und Sozialpolitik die Bearbeitung der Folgeprobleme der zunehmenden Ausdifferenzierung und Entkoppelung einzelner Politikfelder ist, scheint gleichzeitig die Verlagerung der Problemlösung auf eine zentrale Ebene durchaus möglich zu sein und von institutionellen Barrieren nicht notwendigerweise behindert zu werden. Dabei können einzelne erzielte Zwischenergebnisse durchaus einer sektoralen Logik folgen und der Gesamtstrategie widersprechen (wie z.B. die Erweiterung des Vorruhestands in den Niederlanden in der zweiten Hälfte der Achtzigerjahre). Die langfristige Anlage von Beschäftigungspakten ermöglicht hier auch eine nachträgliche Korrektur von sektoralen Abweichungen.

Zweitens scheint die Vermutung bestätigt zu werden, dass es auch unter den Bedingungen eines zunehmenden Regimewettbewerbs der Sozial- und Arbeitsregime in Europa national spezifische Möglichkeiten einer kompensierenden Politik gibt. Diese sind jedoch bestimmten einschränkenden Bedingungen unterworfen. Die Bedingungen, unter denen die Formen einer kompensierenden Politik erkennbar sind, sind zum einen die zunehmende Beschränkung auf die Finanzierung der Dynamisierung der Arbeitsmärkte und weniger auf die Stilllegung des Arbeitsangebots, zum anderen die Beschränkung auf die Umverteilung innerhalb der Arbeitnehmerschaft. Da jedoch auch und gerade innerhalb der Arbeitnehmerschaft Risiken und Möglichkeiten von Beschäftigung zunehmend ungleich verteilt sind, ist dies keine gering einzuschätzende Gestaltungsmacht. 


\section{Literaturverzeichnis}

Alvarez, R. Michael/Garrett, Geoffrey/Lange, Peter, 1991: Government Partisanship, Labor Organization, and Macroeconomic Performance, in: American Political Science Review 85 (2), 539-556.

Aust, Stefan, 1999: The „Celtic Tiger" and its beneficiaries. „Competitive corporatism“ in Ireland. Paper presented at the ECPR Joint Sessions of Workshops in Mannheim, March 26-31.

Barreto, José/Naumann, Reinhard, 1998: Portugal: Industrial Relations under Democracy, in: Anthony Ferner/Richard Hyman (Hrsg.), Changing Industrial Relations in Europe, Oxford, 395-425.

Berthold, Norbert/Hank, Rainer, 1999: Bündnis für Arbeit: Korporatismus statt Wettbewerb, Walter Eucken Institut, Beiträge zur Ordnungstheorie und Ordnungspolitik, Tübingen.

Bispinck, Reinhard, 1997: The Chequered History of the Alliance for Jobs, in: Guiseppe Fajertag/Philippe Pochet (Hrsg.), Social Pacts in Europe, Brussels, 63-78.

Clayton, Richard/Pontusson, Jonas, 1998: Welfare-State Retrenchment Revisited: Entitlement Cuts, Public Sector Restructuring, and Inegalitarian Trends in Advanced Capitalist Societies, in: World Politics, 51 (1), 67-98.

Deutsche Bundesbank, 1999: Der Europäische Arbeitsmarkt und die Währungsunion. Monatsbericht der Deutschen Bundesbank, Oktober, Frankfurt a.M.

Da Paz Ventura Campos Lima, Maria/Naumann, Reinhard, 1997: Social Dialogue and social pacts in Portugal, in: Philippe Pochet/Giuseppe Fajertag (Hrsg.), Social Pacts in Europe, Brüssel, 157-180.

Ebbinghaus, Bernhard/Hassel, Anke, forthcoming: Striking Deals. The role of concertation in the reform of the welfare state, iIn: European Journal of Public Policy.

Ferner, Anthony/Hyman, Richard, 1998: Introduction: Towards European Industrial Relations?, in: Anthony Ferner/Richard Hyman (Hrsg.) Changing Industrial Relations in Europe, Oxford, xi-xxvi.

Freeman, R. B., 1995: The Large Welfare State as a System, in: American Economics Association Papers and Proceedings 85 (2), 16-21.

Hassel, Anke, 1998: Soziale Pakte in Europa, in: Gewerkschaftliche Monatshefte 49 (10), 626-637.

Immergut, Ellen, 1992: The Rules of the Game: The Logic of Health Policy Making in France, Switzerland and Sweden, in: Sven Steinmo/Kathleen Thelen/Frank Longstreth (Hrsg.), Structuring Politics. Historical Institutionalism in Comparative Analysis, Cambridge MA, 57-89.

Karlhofer, Ferdinand/Sickinger, Hubert, 1999, Korporatismus und Sozialpakte im europäischen Vergleich, in: Ferdinand Karlhofer/Emmerich Talos (Hrsg.), Zukunft der Sozialpartnerschaft. Veränderungsdynamik und Reformbedarf, Wien, 241-275.

Kuntze, O.-E. 1998: Neue, korporativistische Einkommenspolitik in europäischen Ländern - Anachronismus oder Standortvorteil?, in: ifo-schnelldienst (34-35), 25-43.

Kommission der Europäischen Gemeinschaften, 1993: Wachstum, Wettbewerbsfähigkeit, Beschäftigung. Herausforderungen der Gegenwart und Wege ins 21. Jahrhundert, Weißbuch, Brüssel.

Kommission der Europäischen Gemeinschaften, 1997: Joint Employment Report 1997 (Rev. 8), Brüssel, 30 September.

Kommission der Europäischen Gemeinschaften, 1998a: Joint Action Programme 1998, Brüssel.

Kommission der Europäischen Gemeinschaften, 1998b: Joint Employment Report 1998, Brüssel.

Lange, Peter, 1984: Unions, Workers and Wage Regulation: The Rational Bases of Consent, in: John H. Goldthorpe (Hrsg.), Order and Conflict in Contemporary Capitalism, Oxford, 98-123.

Mayntz, Renate 1992: Interessenverbände und Gemeinwohl - Die Verbändestudie der Bertelsmann Stiftung, in: Renate Mayntz (Hrsg.), Verbände zwischen Mitgliederinteressen und Gemeinwohl. Gütersloh, 11-35.

OECD, 1997: Implementing the OECD Jobs Strategy. Lessons from member countries' experience, Paris.

O'Donnell, Rory und Colm O'Reardon, 1997: Ireland's experiment in social partnership 1987-96, in: Philippe Pochet/Giuseppe Fajertag (Hrsg.), Social Pacts in Europe, Brüssel, 157-180. 
Oppen, Maria, 1997: Concerted Co-operation and immobilism: labour policy and the regulation of early exit, in: F. Naschold/M. Muramatsu (Hrsg.), State and Administration in Japan and Germany. A Comparative Perspective on Continuity and Change, Berlin/New York, 247-280.

Perez, Sofia, 1999: The Resurgence of National Social Bargaining in Europe: Explaining the Italian and Spanish Experiences, Centro de Estudios Avanzados en Ciencias Sociales, Estudio/Working Paper 1999/130 March.

Pierson, Paul, 1994: Dismantling the Welfare State? Reagan, Thatcher, and the politics of retrenchment, Cambridge.

Pierson, Paul, 1996: The New Politics of the Welfare State, in: World Politics 48 (2), 143-179.

Pizzorno, Alessandro, 1978: Political Exchange and Collective Identity in Industrial Conflict, in: Colin Crouch/Alessandro Pizzorno (Hrsg.): The resurgence of class conflict in western societies since 1968, London.

Pochet, Philippe/Fajertag, Giuseppe, 1997: Social Pacts in Europe in the 1990s. Towards a European social pact?, in: Philippe Pochet/Giuseppe Fajertag (Hrsg.), Social Pacts in Europe, Brüssel, 9-26.

Regalia, Ida/Regini, Marino, 1998: „Italy: The Dual Character of Industrial Relations“, in: Anthony Ferner/Richard Hyman (Hrsg.), Changing Industrial Relations in Europe, Oxford, 459-503.

Regini, Marino, 1999: Between De-regulation and Social Pacts. The Responses of European Economies to Globalization. Centro de Estudios Avanzados en Ciencias Sociales, Estudio/Working Paper 1999/133 March.

Regini, Marino, 1997: Still Engaging in Corporatism? Recent Italian Experience in Comparative Perspective, in: European Journal of Industrial Relations 3 (3) 259-78.

Rhodes, Martin, 1997: Globalisation, Labour Markets and Welfare States. A Future of „Competitive Corporatism“?, in: Martin Rhodes/Yves Mény (Hrsg.), The Future of European Welfare: A New Social Contract?, London, 178-203.

Scharpf, Fritz W., 1991a: Die Handlungsfähigkeit des Staates am Ende des zwanzigsten Jahrhunderts, in: Politische Vierteljahresschrift 32 (4), 621-634.

Scharpf, Fritz W., 1991b: Einführung: Zur Theorie von Verhandlungssystemen, in: Arthur Benz/Fritz W. ScharpflReinhard Zintl (Hrsg.), Horizontale Politikverflechtung. Zur Theorie von Verhandlungssystemen, Frankfurt a.M., 11-27.

Scharpf, Fritz W., 1993, Coordination in Hierarchies and Networks, in: Fritz W. Scharpf(Hrsg.), Games in Hierarchies and Networks, Frankfurt a.M./Boulder, Col., 125-165.

Schmitter, Philippe C./Grote, Jürgen, 1997: Der korporatistische Sysiphus. Vergangenheit, Gegenwart und Zukunft, in: Politische Vierteljahresschrift 38 (3), 530-554.

Schulten, Thorsten, 1998: Tarifpolitik unter den Bedingungen der Europäischen Währungsunion Überlegungen zum Aufbau eines tarifpolitischen Mehrebenensystems am Beispiel der westeuropäischen Metallindustrie, in: WSI-Mitteilungen 51 (7), 482-492.

Streeck, Wolfgang/Schmitter, Philippe (Hrsg.), 1985: Private Interest Government: Beyond Market and State, Beverly Hills/London.

Swenson, Peter, 1992: Union Politics, the Welfare State, Intraclass Conflict in Sweden and Germany, in: M. Golden/J. Pontusson (Hrsg.), Bargaining for Change: Union Politics in North America and Europe, Ithaca.

Telljohann, Volker, 1998: Die Erfahrungen mit tripartistischen Abkommen in Italien, in: Gewerkschaftliche Monatshefte 49 (10), 650-661.

Telljohann, Volker, 1999: Italien: Neue Sozialpakte im Vorfeld der EWU, in: Thorsten Schulten/Reinhard Bispinck (Hrsg.), Tarifpolitik unter dem EURO. Perspektiven einer europäischen Koordinierung: das Beispiel Metallindustrie, Hamburg, 140-167.

Tidow, Stefan, 1998: Europäische Beschäftigungspolitik. Die Entstehung eines neuen Politikfeldes: Ursachen, Hintergründe und Verlauf des politischen Prozesses, Forschungsgruppe Europäische Gemeinschaften (FEG) Arbeitspapier Nr. 18, Philipps-Universität Marburg.

Traxler, Franz, 1995: Farewell to labour market associations? Organized versus disorganized decentralization as a map for industrial relations, in: Colin Crouch/Franz Traxler (Hrsg.), Organized Industrial Relations in Europe: What Future? Aldershot, 3-20. 
Vilrokx, Jacques, 1998: Tarifpolitik am Wendepunkt. Zum Wandel der Tarifvertragsbeziehungen in Belgien in den 90er Jahren, in: WSI-Mitteilungen 51 (7), 478.

Van Wijnbergen, Christa, 1999: The Politics of Reforms: Battling Unemployment in Germany and the Netherlands. Paper prepared for presentation at the 57th Annual Meeting of the Midwest Political Science Associatgion, Chicago, April 15-17.

Visser, Jelle, 1998: Concertation - the Art of Making Social Pacts, in: Emilio Gabaglio/Reiner Hoffmann (Hrsg.), European Trade Union Yearbook, Brüssel, 217-232.

Visser, Jelle/Hemerjick, Anton, 1998: Ein holländisches Wunder. Reform des Sozialstaates und Beschäftigungswachstum in den Niederlande, Frankfurt a.M. 